\title{
Complex hyperbolic lattices
}

\author{
John R. Parker
}

\begin{abstract}
The purpose of this paper is twofold. First, we give a survey of the known methods of constructing lattices in complex hyperbolic space. Secondly, we discuss some of the lattices constructed by Deligne and Mostow and by Thurston in detail. In particular, we give a unified treatment of the constructions of fundamental domains and we relate this to other properties of these lattices.
\end{abstract}

\section{Introduction}

One may construct lattices in complex hyperbolic space in several different ways. Nevertheless, it is often hard to do so and there are relatively few explicit constructions known. The first aim of this paper is to outline the different approaches and give some links between them. Broadly speaking, there are four major constructions: arithmetic constructions, use of moduli of different objects, algebraic geometry and construction of fundamental domains. The second main purpose of this article is to show how these four themes relate to one another for a particular class of lattices, and in particular to give a uniform treatment of recent research in this area. By understanding these lattices at a deeper level, we hope to be able to isolate important features that will enable new lattices to be constructed.

The article is organised as follows. In Section 2, I begin by discussing complex hyperbolic space, lattices and arithmeticity. I have attempted, in Section 2.2, to summarise every construction of complex hyperbolic space that is known. In later sections I specialise to the family of lattices constructed by Deligne, Mostow and Thurston. In Section 3 I discuss the different approaches taken by Deligne and Mostow, Section 3.1, and by Thurston, Section 3.2. Many of these lattices exhibit three-fold symmetry. In Section 4 I discuss the classification and commensurability among Deligne-Mostow-Thurston lattices with three fold symmetry and I also try to show how the different methods of constructing these lattices are related. In particular, in Section 5 I outline some recent constructions of fundamental domains for these lattices. Finally, in Section 6 I give some open problems.

I am very grateful to the people who have read this article and who have given me corrections and suggestions. In particular, I would like to thank Curt McMullen, Domingo Toledo, Frank Johnson and Julien Paupert, as well as the referee.

2000 Mathematics Subject Classification. Primary 22E40. 


\section{Background}

2.1. Complex hyperbolic space. Complex hyperbolic space $\mathbf{H}_{\mathbb{C}}^{n}$ is the natural complex analogue of (real) hyperbolic space $\mathbf{H}^{n}=\mathbf{H}_{\mathbb{R}}^{n}$. The Poincaré disc and halfplane models of the hyperbolic plane are, in fact, complex hyperbolic 1-space $\mathbf{H}_{\mathbb{C}}^{1}$ and so complex hyperbolic space may be regarded as a generalisation of the hyperbolic plane to higher complex dimensions. Background material on complex hyperbolic geometry may be found in the books by Goldman [20] and Schwartz [58]. A more elementary approach with emphasis on discrete groups of isometries may be found in the forthcoming book of Parker [49].

Let $\mathbb{C}^{n, 1}$ be a complex vector space of dimension $n+1$ equipped with a Hermitian form of signature $(n, 1)$. In other words, this form corresponds to a non-singular Hermitian matrix $H$ with $n$ positive eigenvalues and one negative eigenvalue. For column vectors $\mathbf{z}$ and $\mathbf{w}$ in $\mathbb{C}^{n, 1}$, we write the Hermitian form as

$$
\langle\mathbf{z}, \mathbf{w}\rangle=\mathbf{w}^{*} H \mathbf{z} \text {. }
$$

Here $\mathbf{w}^{*}$ is the Hermitian transpose of $A$, that is the row vector which is the transpose of the matrix whose entries are the complex conjugates of the entries of w. Let $\mathrm{U}(H)$ denote the group of $(n+1) \times(n+1)$ complex matrices that are unitary with respect to $H$. That is, $A \in \mathrm{U}(H)$ if and only if $A^{*} H A=H$ and so $A^{-1}=H^{-1} A^{*} H$. Once again $A^{*}$ is the Hermitian transpose of $A$. Let $\mathrm{SU}(H)$ be the subgroup of $\mathrm{U}(H)$ comprising matrices with determinant +1 . For results that do not depend on the particular form used but only on the signature $(n, 1)$ we will write $\mathrm{U}(n, 1)$ and $\mathrm{SU}(n, 1)$, respectively.

If $\mathbf{z} \in \mathbb{C}^{n, 1}$ then $\langle\mathbf{z}, \mathbf{z}\rangle=\mathbf{z}^{*} H \mathbf{z}$ is real. Let $V_{-}, V_{0}$ and $V_{+}$be the subsets of $\mathbb{C}^{n, 1}-\{0\}$ consisting of vectors where $\langle\mathbf{z}, \mathbf{z}\rangle$ is negative, zero or positive respectively. There is a natural complex projection $\mathbb{P}$ from $\mathbb{C}^{n, 1}-\{0\}$ to $\mathbb{C P}^{n}$ obtained by identifying all non-zero complex multiples of a given vector. Since

$$
\langle\lambda \mathbf{z}, \lambda \mathbf{z}\rangle=(\lambda \mathbf{z})^{*} H(\lambda \mathbf{z})=|\lambda|^{2} \mathbf{z}^{*} H \mathbf{z}=|\lambda|^{2}\langle\mathbf{z}, \mathbf{z}\rangle
$$

for $\lambda \in \mathbb{C}-\{0\}$, we see that if $\mathbf{z}$ is in $V_{-}, V_{0}$ or $V_{+}$then so is $\lambda \mathbf{z}$. Hence the projection map $\mathbb{P}$ respects $V_{-}, V_{0}$ and $V_{+}$. The projective model of complex hyperbolic space is $\mathbf{H}_{\mathbb{C}}^{n}=\mathbb{P} V_{-}$and $\partial \mathbf{H}_{\mathbb{C}}^{n}=\mathbb{P} V_{0}$. The metric on $\mathbf{H}_{\mathbb{C}}^{n}$ is the Bergman metric, given by

$$
d s^{2}=\frac{-4}{\langle\mathbf{z}, \mathbf{z}\rangle^{2}} \operatorname{det}\left(\begin{array}{cc}
\langle\mathbf{z}, \mathbf{z}\rangle & \langle d \mathbf{z}, \mathbf{z}\rangle \\
\langle\mathbf{z}, d \mathbf{z}\rangle & \langle d \mathbf{z}, d \mathbf{z}\rangle
\end{array}\right) .
$$

The factor -4 means that the sectional curvatures of $\mathbf{H}_{\mathbb{C}}^{2}$ are pinched between -1 and $-1 / 4$. Other authors use a different constant and so obtain other curvatures.

The holomorphic isometry group of $\mathbf{H}_{\mathbb{C}}^{n}$ is $\mathrm{PU}(H)=\mathrm{U}(H) /\left\{e^{i \theta} I: \theta \in[0,2 \pi)\right\}$. The full isometry group of $\mathbf{H}_{\mathbb{C}}^{n}$ is generated by $\mathrm{PU}(H)$ and complex conjugation. Clearly there are only $n+1$ matrices in $\mathrm{SU}(H)$ of the form $e^{i \theta} I$ and for these matrices $\theta=2 \pi k /(n+1)$ for $k=0, \ldots, n$. Thus $\mathrm{SU}(H)$ is an $(n+1)$-fold cover of $\mathrm{PU}(H)$. This generalises the well known fact that the group of unimodular $2 \times 2$ matrices is the double cover of the corresponding Möbius group.

A lattice in a locally compact topological group $G$ with Haar measure is a discrete subgroup $\Gamma$ of $G$ so that the quotient $\Gamma \backslash G$ has finite volume. In particular, when $G$ is $\mathrm{PU}(H)$ then a lattice is a discrete subgroup $\Gamma$ of $\mathrm{PU}(H)$ so that the quotient $\Gamma \backslash \mathbf{H}_{\mathbb{C}}^{n}$ has finite volume with respect to the Bergman metric. A lattice $\Gamma$ in $\mathrm{PU}(H)$ is called uniform or cocompact if the quotient $\Gamma \backslash \mathbf{H}_{\mathbb{C}}^{n}$ is compact and is called non-uniform or cofinite-volume otherwise. 
I will conclude this section by discussing the relationship between arithmetic groups and lattices. This provides motivation for the study of complex hyperbolic lattices. I will discuss arithmeticity, and give a definition, in the next section.

Like real hyperbolic space, complex hyperbolic space is an example of a rank 1 symmetric space of non-compact type. The other rank 1 symmetric spaces of noncompact type are quaternionic hyperbolic space $\mathbf{H}_{\mathbb{H}}^{n}$ and the octonionic hyperbolic plane $\mathbf{H}_{\mathbb{O}}^{2}$; see Chapter 19 of Mostow's book [40]. (Quaternionic hyperbolic 1-space $\mathbf{H}_{\mathbb{H}}^{1}$ is canonically identified with $\mathbf{H}_{\mathbb{R}}^{4}$ and octonionic hyperbolic 1-space $\mathbf{H}_{\mathbb{O}}^{1}$ with $\mathbf{H}_{\mathbb{R}}^{8}$.)

A fundamental problem in the study of symmetric spaces is the relationship between arithmetic groups and lattices. On one hand, Borel and Harish-Chandra [9] proved that in all symmetric spaces of non-compact type all arithmetic groups are lattices. On the other hand, Margulis [36] showed that when the rank is at least 2 then all irreducible lattices are arithmetic. Likewise, Corlette [10] and Gromov and Schoen [22] have shown that in $\mathbf{H}_{\mathbb{H}}^{n}$ for $n \geq 2$ and in $\mathbf{H}_{\mathbb{O}}^{2}$ all lattices are arithmetic. Furthermore, Gromov and Piatetski-Shapiro [21] have given examples of non-arithmetic lattices in $\mathbf{H}_{\mathbb{R}}^{n}$ for all $n \geq 2$. Complex hyperbolic space is the only class of symmetric spaces of non-compact type where this question has not been settled. Mostow [41] constructed examples of non-arithmetic lattices in $\mathbf{H}_{\mathbb{C}}^{2}$ and we shall discuss these examples below. Deligne and Mostow [11] found a nonarithmetic lattice in $\mathbf{H}_{\mathbb{C}}^{3}$. For $\mathbf{H}_{\mathbb{C}}^{n}$ with $n \geq 4$ the question is open and probably represents the most important open question in complex hyperbolic geometry. The fact that there are relatively few known constructions of complex hyperbolic lattices may well account for the fact that this problem is still open. This gives some motivation for studying complex hyperbolic lattices.

2.2. Methods of constructing lattices. In this section we outline the methods of construction of complex hyperbolic lattices that are known. As indicated in the introduction, these broadly fall into four main categories. Inevitably there is some overlap between these, and the same lattice may be viewed from different points of view. Indeed, the purpose of many of the papers listed below is to use a new technique to describe a lattice that is already known and therefore to obtain new information about it. Other papers listed below show that lattices previously constructed by very different methods are actually the same. Many of the constructions listed below have been known for some time and I have given early references where I am aware of them.

Before beginning this survey, there are two observations I would like to make. First, I have attempted to make this section as wide ranging as possible, but inevitably there will be references I have missed. The bibliographies of the papers listed below should fill the gaps I have left. Secondly, this discussion is quite superficial and I refer the reader to the cited papers for detailed definitions and statements of theorems. In the case of Deligne-Mostow lattices in $\mathrm{PU}(2,1)$ many of the details of the different descriptions may be found in later sections of this paper.

The first technique involves using number theory to construct arithmetic lattices. The natural inclusion of the integers in the real numbers is the most familiar discrete subset of a continuous object. This may be extended to the inclusion of the non-uniform lattice $\operatorname{PSL}(2, \mathbb{Z})$ in $\operatorname{PSL}(2, \mathbb{R})$ with its natural action on the upper half plane model of $\mathbf{H}_{\mathbb{C}}^{1}$. This construction was generalised by Picard [52] in 1883 
and also in $[\mathbf{5 3}]$ a year later. Let $d$ be a positive square-free integer and $\mathbb{Q}(i \sqrt{d})$ be the corresponding quadratic imaginary number field. The ring of integers $\mathcal{O}_{d}$ in $\mathbb{Q}(i \sqrt{d})$ is either $\mathbb{Z}[i \sqrt{d}]$ if $d \equiv 1,2(\bmod 4)$ or $\mathbb{Z}\left[\frac{1+i \sqrt{d}}{2}\right]$ if $d \equiv 3(\bmod 4)$. We can easily see that $\mathcal{O}_{d}$ is a discrete subring of $\mathbb{C}$. Suppose that $H$ is a Hermitian matrix of signature $(2,1)$ with entries in $\mathcal{O}_{d}$. Let $\mathrm{SU}\left(H ; \mathcal{O}_{d}\right)$ denote the subgroup of $\mathrm{SU}(H)$ consisting of those matrices whose entries lie in $\mathcal{O}_{d}$. Then it is clear that $\mathrm{SU}\left(H ; \mathcal{O}_{d}\right)$ is a discrete subgroup of $\mathrm{SU}(H)$ and a little more work shows that it is a non-uniform lattice. In [52], Picard considered the case where $H$ is the diagonal matrix with entries $(1,1,-1)$ and he studied $\mathrm{PU}\left(H ; \mathcal{O}_{d}\right)$ acting on $\mathbf{H}_{\mathbb{C}}^{2}$. These groups, called Picard modular groups, were further studied by Alezais [1] who obtained generators for $\mathrm{SU}\left(H ; \mathcal{O}_{3}\right)$. More recently Holzapfel $[\mathbf{2 6}],[\mathbf{2 7}]$ has studied these groups in great detail, using a combination of arithmetic methods and algebraic geometry. The geometry of the group $\mathrm{SU}\left(H ; \mathcal{O}_{3}\right)$ has been studied by Falbel and Parker [17] and the geometry of $\mathrm{SU}\left(H ; \mathcal{O}_{1}\right)$ has been studied by by Francsics and Lax [18] and Falbel, Francsics and Parker [16]. There is an obvious generalisation of Picard modular groups to higher complex dimensions.

These groups are examples of arithmetic groups; see Borel and Harish-Chandra [9] or Chapter X of Raghunathan [56]. A linear algebraic group defined over $\mathbb{Q}$ is a matrix group $G \subset \mathrm{GL}(m, \mathbb{C})$ that consists of all invertible matrices whose coefficients satisfy some set of polynomial equations on $\mathrm{M}(m, \mathbb{C})$ with rational coefficients. Let $G_{\mathbb{Z}}$ be the intersection of $G$ with $\operatorname{GL}(m, \mathbb{Z})$ and $G_{\mathbb{R}}$ be the intersection of $G$ with $\mathrm{GL}(m, \mathbb{R})$. Then $G_{\mathbb{Z}}$ is an arithmetic subgroup of $G_{\mathbb{R}}$. Let $\phi: G_{\mathbb{R}} \longrightarrow \mathrm{SU}(H)$ be a continuous, surjective homomorphism with compact kernel. Then $\Gamma<\mathrm{SU}(H)$ is said to be arithmetic if $\Gamma$ is commensurable with $\phi\left(G_{\mathbb{Z}}\right)$. See the notes by McReynolds [38] for more details about arithmetic subgroups of $\mathrm{SU}(H)$.

Arithmetic lattices have been constructed by a variety of authors. For example Mumford [45] used $p$-adic uniformization to construct a fake projective plane, that is, the quotient of $\mathbf{H}_{\mathbb{C}}^{2}$ by a torsion free uniform lattice in $\mathrm{PU}(H)$ with Euler characteristic 3. Two more examples were constructed by Ishida and Kato [31]. Recently Prasad and Yeung [55] have given a classification of possible fake projective planes.

Another recent example is due to Deraux [14], who used an arithmetic construction to construct a lattice from a triangle group by imposing an extra relation. The number field Deraux uses is on the list given by Prasad and Yeung [55]. Parker and Thompson [51] have constructed a fundamental domain for Deraux's lattice and have shown that the Euler characteristic of its quotient orbifold is the same as the maximal group over the same number field obtained by Prasad and Yeung in $[55]$.

The second major technique for constructing complex hyperbolic lattices is to consider objects that are parametrised by complex hyperbolic space with the property that the corresponding automorphism group is a complex hyperbolic lattice. This generalises the well known fact that the upper half plane $\mathbf{H}_{\mathbb{C}}^{1}$ is the projectivisation of the parameter space of lattices in $\mathbb{C}$ with a prescribed basis, and different bases for the same lattice are related by an element of $\operatorname{PSL}(2, \mathbb{Z})$. Thus $\operatorname{PSL}(2, \mathbb{Z})$ is the monodromy group of elliptic functions. Moreover, $\mathbf{H}_{\mathbb{C}}^{1}$ is the Teichmüller space of the punctured torus and $\operatorname{PSL}(2, \mathbb{Z})$ is the mapping class group, and so is also the Teichmüller modular group. Similarly, Schwarz [59] showed that any hyperbolic triangle group can arise the monodromy group of a hypergeometric function. The 
first examples of this type of construction for higher complex dimensions are, once again, due to Picard [54]. He considered the moduli space of certain multi-variable hypergeometric functions and showed that their monodromy groups were lattices in $\mathrm{PU}(2,1)$. These functions were subsequently studied by Lauricella [33]. Le Vavasseur [34] gave a complete list of 27 groups that satisfied Picard's hypotheses. Picard's proof of discreteness was not complete and this mistake was corrected by Deligne and Mostow [11]. The 27 groups on Le Vavasseur's list and the 7 similar examples in $\mathrm{PU}(3,1)$ and one in each of $\mathrm{PU}(4,1)$ and $\mathrm{PU}(5,1)$ are described in detail by Terada [62] and by Deligne and Mostow [11]. Picard's criterion is called INT by Deligne and Mostow [11]. In [70] Yoshida associated Coxeter graphs to each of the 27 lattices described by Le Vavasseur, see Figure 1 and the related discussion below.

The criterion INT implies that the monodromy group is discrete. Mostow then asked about the discreteness of monodromy groups that fail condition INT. In [42] he relaxed Picard's criterion to obtain a condition $\Sigma$ INT which applies to monodromy groups with symmetry. In [44] Mostow discussed the groups satisfying $\Sigma$ INT. An alternative but equivalent approach to these lattices was given by Thurston [63] who gave a list of all 94 monodromy groups that satisfy $\Sigma I N T$ and so give lattices in $\mathrm{PU}(n, 1)$. (This list also appeared in [44].) The values of $n$ range between 2 and 9. Thurston's idea was to consider the space of Euclidean cone metrics on the sphere with prescribed cone angles, that is, Euclidean polyhedra with prescribed total angle at the vertices. The branch points of the hypergeometric functions correspond to the vertices of the polyhedra. Details of Thurston's construction were given by Weber [66] in his thesis and explicit constructions have been given by Parker [47] for Livné's lattices and Boadi [8] for some of Mostow's lattices. Thurston's approach was generalised to other surfaces by Veech [65]. Special cases of the Deligne-Mostow and Thurston construction have recently appeared in work of McMullen [37] in connection with his investigation into moduli space and unitary representations of braid groups.

Furthermore, in [44] Mostow investigated precisely which monodromy groups lead to discrete lattices. He showed that for $n \geq 4$ the criterion $\Sigma$ INT precisely characterises discreteness and when $n=3$ there is exactly one discrete monodromy group that fails $\Sigma$ INT. The situation for $n=2$ is more complicated. With nine exceptions, Mostow was able to prove that all monodromy groups are either nondiscrete or satisfy $\Sigma$ INT. In [57] Sauter then showed that each of these nine monodromy groups in $\mathrm{PU}(2,1)$ is commensurable with a monodromy group satisfying $\Sigma$ INT and hence is discrete. In their book [12] Deligne and Mostow extended Sauter's work on commensurability. In Section 3.3 below we discuss these commensurability theorems. Further connections between these lattices are given by Toledo [64] who lists all holomorphic maps between various orbifolds corresponding to lattices satisfying $\Sigma$ INT.

There are other examples of complex hyperbolic lattices that arise as automorphism groups of geometrical objects. Allcock [2] has constructed complex hyperbolic lattices in $\mathrm{PU}(5,1), \mathrm{PU}(9,1)$ and $\mathrm{PU}(13,1)$ by considering the automorphism groups of Lorentzian lattices over the Eisenstein integers $\mathcal{O}_{3}$, the largest example coming from the Leech lattice. Allcock's lattice in $\mathrm{PU}(9,1)$ is the same as the one given by Deligne and Mostow [11]. In [3], Allcock used a related construction to give several examples of lattices, including examples in $\mathrm{PU}(4,1)$ and $\operatorname{PU}(7,1)$ 
which do not appear on the list of Deligne and Mostow [11]. Furthermore, Allcock, Carlson and Toledo [4] show that the moduli space of cubic complex surfaces is isomorphic to the quotient of $\mathbf{H}_{\mathbb{C}}^{4}$ by one of the lattices constructed in [3]. The same three authors have also shown [5] that the moduli space of cubic complex three manifolds is isomorphic to the quotient of $\mathbf{H}_{\mathbb{C}}^{10}$ by a lattice.

One may also use algebraic geometry to construct complex hyperbolic lattices. The uniformization theorem of Yau, later extended by Miyaoka, states that if $M$ is a compact complex 2-manifold whose Chern classes satisfy $c_{1}^{2}(M)=3 c_{2}(M)$ then either $M$ is $\mathbb{C P}^{2}$ or $M$ is the quotient of the unit ball in $\mathbb{C}^{2}$ by a group of biholomorphisms. In other words, in the latter case, $M$ is the quotient of $\mathbf{H}_{\mathbb{C}}^{2}$ by a uniform lattice in $\mathrm{PU}(2,1)$. This theorem was originally proved by Yau [68] for manifolds with ample canonical bundle. Later the restriction on the canonical bundle was removed by Miyaoka, Theorem 4 of [39].

The Yau-Miyaoka uniformization theorem enables complex hyperbolic lattices to be constructed using algebraic geometry. It may be thought of as a generalisation of the well known fact that there is a natural correspondence between complex algebraic curves and Riemann surfaces. It is generally not straightforward to pass between these two descriptions. The first explicit examples of complex hyperbolic lattices arising from this construction are due to Livné [35]. Subsequently, more examples were given by Hirzebruch $[\mathbf{2 4}]$ and [25] and Shvartsman [60]; see also the survey $[\mathbf{2 3}]$ and the book $[\mathbf{6}]$. The connections between the constructions of Livné and Hirzebruch is discussed in [30]. These examples involve line arrangements. Consider $k$ distinct complex lines $L_{1}, \ldots, L_{k}$ in $\mathbb{C P}^{2}$. One may then construct an algebraic surface branched along each of the lines $L_{j}$. It is clear that the groups constructed by Deligne-Mostow and Thurston also fit into this general scheme. Here the $L_{j}$ are the complex lines where two of the cone points collide. We shall give the associated line arrangements when we discuss the Deligne-Mostow-Thurston groups. Sauter uses these line arrangements to calculate the Euler characteristic of these orbifolds $[\mathbf{5 7}]$.

In [67] Yamazaki and Yoshida relate Hirzebruch's examples to hypergeometric functions. In [61] Shvartsman used algebraic geometry to describe one of the nonarithmetic lattices from Deligne and Mostow [11] and a combination of algebraic geometry and arithmetic has been used by Holzapfel [28]. Techniques from algebraic geometry are used alongside arithmetic methods in the construction of fake projective planes by Mumford [45] and Prasad and Yeung [55] described above.

The final method of constructing a complex hyperbolic lattice $\Gamma$ in $\operatorname{PU}(n, 1)$ is to find a fundamental domain for its action on $\mathbf{H}_{\mathbb{C}}^{n}$. In other words, one must find an open connected set $D \subset \mathbf{H}_{\mathbb{C}}^{n}$ so that $D \cap A(D)=\emptyset$ for all $A \in \Gamma-\{I\}$ and $\bigcup_{A \in \Gamma} A(\bar{D})=\mathbf{H}_{\mathbb{C}}^{n}$, where $\bar{D}$ is the closure of $D$ inside $\mathbf{H}_{\mathbb{C}}^{n}$. There are no totally geodesic real hypersurfaces in $\mathbf{H}_{\mathbb{C}}^{n}$ for $n \geq 2$. This makes the construction of a fundamental domain $D$ rather more complicated than for spaces of constant curvature.

Typically, a fundamental domain is a locally finite polyhedron $D$ with some combinatorial structure. The codimension one faces of $D$, called sides, may be contained in a wide variety of real hypersurfaces, but there should exist a set of side pairing maps: each side should be mapped bijectively to another side (possibly itself) by a map $A$ in $\mathrm{PU}(n, 1)$. Given such data, Poincaré's polyhedron theorem 
gives conditions under which the group generated by the side pairing maps is discrete with $D$ as a fundamental domain; see for example Theorem 6.3 .1 of [41], or Theorem 5.7 of [17]. Moreover, Poincaré's theorem also gives a presentation for this group. As we just indicated, the generators are the side pairing maps and there are two kinds of relations. First, if $A$ maps a side to itself then $A^{2}$ is the identity and the resulting relation is a reflection relation. Secondly, each codimension-2 face of $D$ is contained in two sides. Its images under the side pairing maps corresponding to these two sides are also codimension-2 faces. By iterating, one obtains a cycle of side pairing maps that sends a given codimension-2 face to itself. Some power of this cycle is the identity and gives rise to a cycle relation. Poincaré's theorem asserts that all relations in the group may be obtained from the reflection relations and cycle relations. A further consequence of Poincaré's theorem is that one may obtain the orbifold Euler characteristic of the quotient and so, using the complex hyperbolic Gauss-Bonnet theorem, calculate its volume. We give examples of how to do this below.

One method of building fundamental domains is to construct the Dirichlet domain based at $z_{0} \in \mathbf{H}_{\mathbb{C}}^{n}$. Assuming that $z_{0}$ is not fixed by any non-trivial element of $\Gamma$, the Dirichlet domain $D_{\Gamma}\left(z_{0}\right)$ based at $z_{0}$ is the set of points in $\mathbf{H}_{\mathbb{C}}^{n}$ that are closer to $z_{0}$ that to any other point in the $\Gamma$ orbit of $z_{0}$. The faces of $D_{\Gamma}\left(z_{0}\right)$ are contained in bisectors, that is the locus of points equidistant from a pair of points. Complex hyperbolic Dirichlet domains were constructed by Giraud [19] (see also Appendix A of Goldman $[\mathbf{2 0}])$.

In his famous paper [41], Mostow constructed non-arithmetic complex hyperbolic lattices by building fundamental polyhedra whose sides are contained in bisectors. There were some minor errors in Mostow's construction; see Deraux [13]. An alternative construction of fundamental domains for the same groups was given by Deraux, Falbel and Paupert [15]. We shall discuss their construction in detail below. A related construction for Livné's lattices was given by Parker [47]. The method of $[\mathbf{4 7}]$ was followed by Boadi $[\mathbf{8}]$ for the Mostow lattices not treated in [15]. A major aim of this paper is to show that the constructions of $[\mathbf{1 5}],[\mathbf{4 7}]$ and $[\mathbf{8}]$ are very closely related and to show that, together with commensurability theorems of Sauter [57] and Deligne and Mostow [12], these constructions are sufficient to give detailed information about all Deligne-Mostow-Thurston lattices in $\mathrm{PU}(2,1)$ with three-fold symmetry. Further explicit constructions of fundamental domains have been given by Falbel and Parker for $\mathrm{PU}\left(2,1 ; \mathcal{O}_{3}\right)$, by Francsics and Lax $[\mathbf{1 8}]$ and Falbel, Francsics and Parker $[\mathbf{1 6}]$ for $\mathrm{PU}\left(2,1 ; \mathcal{O}_{1}\right)$ and by Parker and Thompson [51] for Deraux's lattice. The fundamental domains constructed in [8], $[\mathbf{1 5}],[\mathbf{1 7}],[\mathbf{1 6}],[\mathbf{4 7}]$ are all generalisations of Dirichlet domains in the following sense. Instead of taking $z_{0}$ to be a point with trivial stabiliser in $\Gamma$, one takes it to have a larger stabiliser, denoted $\Gamma_{0}$. One then forms the Dirichlet domain for all elements of $\Gamma-\Gamma_{0}$ and intersects it with a fundamental domain for $\Gamma_{0}$. Since the Dirichlet domain is star-like about $z_{0}$ we use a coning process to produce the fundamental domain of $\Gamma_{0}$. Its sides are then foliated by arcs of geodesics through $z_{0}$. This construction is used in [15].

A natural generalisation of the Dirichlet domain is the Ford domain, see Section 9.3 of $[\mathbf{2 0}]$. Here the point $z_{0}$ lies on $\partial \mathbf{H}_{\mathbb{C}}^{n}$ and the distance is replaced with a Busemann function based at $z_{0}$. The level sets of a Busemann function are horospheres. The sides of the Ford domain are contained in bisectors called isometric 
spheres which are the locus where the given element of $\mathrm{PU}(n, 1)$ acts isometrically on $\partial \mathbf{H}_{\mathbb{C}}^{n}-\left\{z_{0}\right\}$ with respect to the Cygan metric, a natural metric on the Heisenberg group. The stabiliser $\Gamma_{0}$ of $z_{0}$ is infinite, nevertheless one may again obtain a fundamental domain for $\Gamma$ by intersecting the Ford domain for $\Gamma-\Gamma_{0}$ with a fundamental domain for $\Gamma_{0}$. This construction is used in [17] and [16], the fundamental domain for $\Gamma_{0}$ is again produced using a coning process over $z_{0}$.

A further generalisation of this process is to take a complex line $L_{0}$ instead of a point $z_{0}$. Let $\Gamma_{0}$ denote the stabiliser of $L_{0}$ inside $\Gamma$. If the image of $L_{0}$ under an element of $\Gamma-\Gamma_{0}$ is disjoint from $L_{0}$ then the locus of points equidistant from these two complex lines is a bisector (otherwise it is a fan or a Clifford cone). When $L_{0}$ does not intersect any of its images under $\Gamma-\Gamma_{0}$ we can form a Dirichlet type domain and then intersect it with a fundamental domain for $\Gamma_{0}$. This is carried out in $[47]$.

\section{Deligne-Mostow-Thurston lattices}

In this section we outline the general constructions of Deligne and Mostow [11], [42], [44] and Thurston [63] of lattices arising as monodromy groups of hypergeometric functions or, equivalently, modular groups of flat cone metrics on the sphere. There is a very nice survey article $[\mathbf{4 3}]$ by Mostow that outlines this construction and gives some of its history.

3.1. Monodromy of hypergeometric functions. Define a ball $N$-tuple $\mu=\left(\mu_{1}, \ldots, \mu_{N}\right)$ to be a set of $N$ real numbers satisfying:

$$
\sum_{j=1}^{N} \mu_{j}=2, \quad 0<\mu_{j}<1 \quad \text { for } j=1, \ldots, N .
$$

Suppose that $\mu=\left(\mu_{1}, \ldots, \mu_{N}\right)$ is a ball $N$-tuple and let $\left(z_{1}, \ldots, z_{N}\right)$ be $N$ distinct points in $\widehat{\mathbb{C}}$. For each pair of distinct $a, b \in\{1, \ldots, N\}$ we can define the hypergeometric function

$$
F_{a b}\left(z_{1}, \ldots, z_{N}\right)=\int_{z=z_{a}}^{z_{b}} \prod_{k=1}^{N}\left(z-z_{k}\right)^{-\mu_{k}} d z
$$

where, apart from its end points, the path of integration lies in $\widehat{\mathbb{C}}-\left\{z_{1}, \ldots, z_{N}\right\}$. If one of the $z_{j}=\infty$ for some $j$ then we omit the corresponding term $\left(z-z_{j}\right)^{-\mu_{j}}$ from the product. Notice that $\operatorname{PSL}(2, \mathbb{C})$ acts by Möbius transformations on the space of variables and so we can define an equivalence relation via

$$
F_{a b}\left(z_{1}, \ldots, z_{N}\right) \sim F_{a b}\left(w_{1}, \ldots, w_{N}\right)
$$

if and only if there exists $A \in \operatorname{PSL}(2, \mathbb{C})$ so that $w_{j}=A\left(z_{j}\right)$ for each $j=1, \ldots, N$. We write $\left[z_{1}, \ldots, z_{N}\right]$ for the equivalence class containing $\left(z_{1}, \ldots, z_{N}\right)$. We may take a canonical representative of this class by setting $z_{N-2}=0, z_{N-1}=1$ and $z_{N}=\infty$. Hence, up to Möbius equivalence, we obtain a function in $N-3$ variables $z_{1}, \ldots, z_{N-3}$.

Following Deligne and Mostow [11] we define $Q$ to be the configuration space of $N$ distinct points on the Riemann sphere up to $\operatorname{PSL}(2, \mathbb{C})$ equivalence. That is,

$$
Q=\left\{\left[z_{1}, \ldots, z_{N}\right] \in \widehat{\mathbb{C}}^{N}: z_{i} \neq z_{j} \text { for } i \neq j\right\} / \operatorname{PSL}(2, \mathbb{C}) .
$$



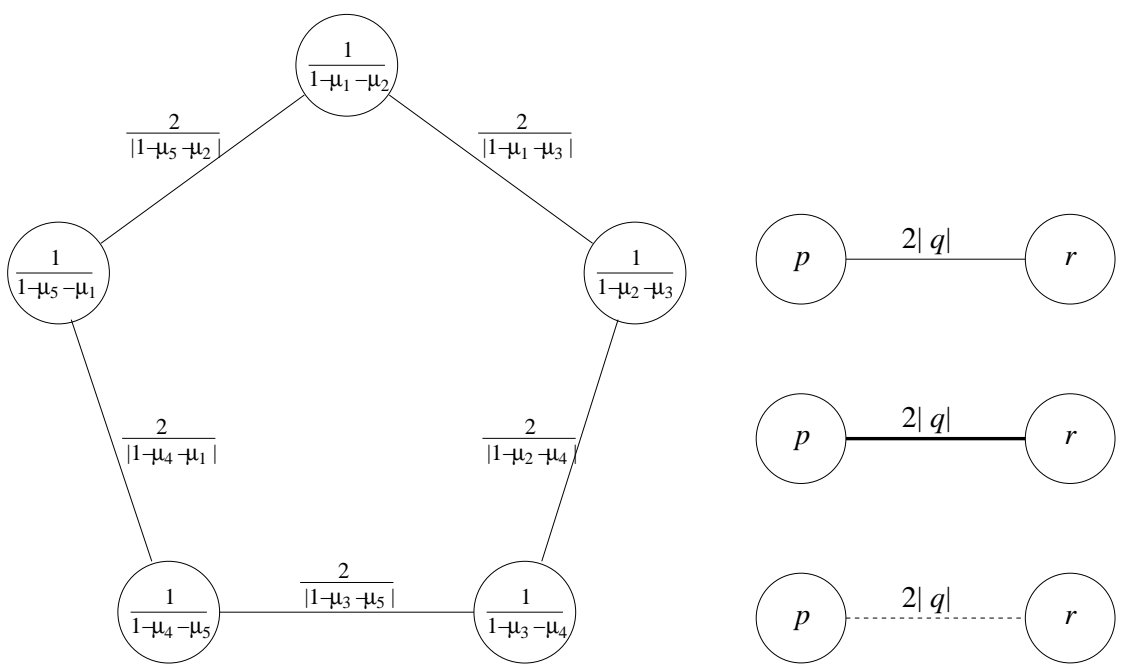

Figure 1. On the left is Yoshida's graph for the quintuple $\left(\mu_{1}, \mu_{2}, \mu_{3}, \mu_{4}, \mu_{5}\right)$. The edges are drawn using different styles of lines as indicated on the right. From top to bottom the different cases are $1 /|p|+1 /|q|+1 /|r|>1,=1,<1$ respectively.

According to [11], the functions $F_{a b}$ form a vector space of dimension $N-2$ and we consider the corresponding projective space, which we canonically identify with $\mathbb{C P}^{N-3}$. Moving along a loop around one of the points $z_{j}$ defines a linear map on the vector space of the $F_{a b}$ and hence a map in $\operatorname{PGL}(N-2, \mathbb{C})$ acting on $\mathbb{C P}^{N-3}$. Each $F_{a b}$ is a multivalued map defined on $Q$ and we may lift this to a single valued map on $\widetilde{Q}$, the universal cover of $Q$. This enables us to construct a single valued map $\omega_{\mu}$ from $\widetilde{Q}$ to $\mathbb{C P}^{N-3}$ which is equivariant with respect to $\pi_{1}(Q)$. Hence $\omega_{\mu}$ induces an representation of $\pi_{1}(Q)$ to $\Gamma_{\mu}<\operatorname{PGL}(N-2, \mathbb{C})$, called the monodromy action. Furthermore, the condition (3.1) implies that there is a Hermitian form of signature $(N-3,1)$ on $\widetilde{Q}$ preserved by this action. Therefore, the monodromy action $\Gamma_{\mu}$ corresponding to $\omega_{\mu}$ lies in $\mathrm{PU}(N-3,1)$. In $[\mathbf{4 2}]$, Mostow gives a criterion called $\Sigma$ INT on ball $N$-tuples which implies that the image of the monodromy representation is a lattice in $\mathrm{PU}(N-3,1)$. Let $\Sigma$ be the subgroup of $S_{N}$ acting on $Q$ so that for each $\sigma \in \Sigma$ then $\sigma\left(z_{j}\right)=z_{k}$ only if $\mu_{j}=\mu_{k}$. In other words, $\Sigma$ freely permutes marked points with the same weight. Let $Q^{\prime}$ be the subset of $Q$ where $\Sigma$ acts without fixed points. The monodromy map can be extended to $Q^{\prime} / \Sigma$ and we let $\Gamma_{\mu \Sigma}$ denote the image of the monodromy representation of $\pi_{1}\left(Q^{\prime} / \Sigma\right)$.

Definition 3.1. Let $\mu=\left(\mu_{1}, \ldots, \mu_{N}\right)$ be a ball $N$-tuple satisfying (3.1). Then $\mu$ is said to satisfy the condition $\Sigma$ INT provided that there is a subset $S$ of $\{1, \ldots, N\}$ so that for any pair $\mu_{j}, \mu_{k}$ with $\mu_{j}+\mu_{k}<1$ either

(i) $1-\mu_{j}-\mu_{k}=1 / n_{j k}$ where $n_{j k} \in \mathbb{Z}$, or

(ii) if $j, k \in S$ then $\mu_{j}=\mu_{k}$ and $1 / 2-\mu_{j}=1 / m_{j k}$ where $m_{j k} \in \mathbb{Z}$.

Theorem 3.2 (Mostow [42]). Let $\mu$ be a ball $N$-tuple satisfying $\Sigma I N T$. Then the associated monodromy representation $\Gamma_{\mu \Sigma}$ is a lattice in $\mathrm{PU}(N-3,1)$. 
The condition $\Sigma$ INT generalises the condition INT given by Deligne and Mostow [11] which characterises Le Vavasseur's 27 lattices [34], see also Terada [62]. In condition INT the quantity $1 /\left(1-\mu_{j}-\mu_{k}\right)$ is required to be an integer for all $j \neq k$ with $0<\mu_{j}+\mu_{k}<1$. To each ball quintuple $\left(\mu_{1}, \ldots, \mu_{5}\right)$ satisfying INT, Yoshida [70] associated a Coxeter-type graph. This is a pentagon with labelled vertices and edges; see Figure 1. The vertices are labelled with the integer $1 /\left(1-\mu_{i}-\mu_{i+1}\right)$ with indices taken cyclically. The edge joining the vertices with labels $1 /\left(1-\mu_{i-1}-\mu_{i}\right)$ and $1 /\left(1-\mu_{i}-\mu_{i+1}\right)$ is labelled with $2 /\left|1-\mu_{i-1}-\mu_{i+1}\right|$. Moreover, Yoshida has different ways of drawing each edge to indicate whether the corresponding triangle group is spherical, fixing a point of $\mathbf{H}_{\mathbb{C}}^{2}$; is Euclidean, fixing a point of $\partial \mathbf{H}_{\mathbb{C}}^{2}$ or is hyperbolic, acting as a Fuchsian subgroup on a complex line.

The monodromy groups $\Gamma_{\mu \Sigma}$ constructed above are clearly related to the mapping class group of the sphere with $N$ marked points. As is well known this mapping class group is closely related to the spherical braid group on $N$ strings; see Birman [7]. The relationship between braid groups and monodromy groups is discussed in detail in Mostow's survey [43] and on pages 336 to 339 of Sauter [57]. This mapping class group is generated by Dehn twists along curves passing through a pair of the marked points. Performing a single Dehn twist swaps the points and its square is a non-trivial self homeomorphism of the punctured sphere that maps each marked point to itself. Because our marked points are not (in general) punctures but the holonomy around them is finite, the monodromies corresponding to these Dehn twists have (in general) finite order.

There is a further difference. It is usual to distinguish between the full mapping class group, where one allows the marked points to be permuted, and the pure mapping class group, where one insists that each marked point is sent to itself. The groups we shall be interested in fall between these two extremes. Namely, we only allow the permutations in the subgroup $\Sigma$ of $S_{N}$ described above. Suppose $z_{j}$ and $z_{k}$ are two of the marked points with associated weights $\mu_{j}$ and $\mu_{k}$. If $\mu_{j} \neq \mu_{k}$ then we are not allowed to interchange $z_{j}$ and $z_{k}$ but if $\mu_{j}=\mu_{k}$ then we are allowed to interchange them.

3.2. Shapes of polyhedra. In [63] Thurston gave an alternative viewpoint on the ball $N$-tuples considered by Deligne and Mostow and described in Section 3.1 .

A cone singularity of a surface is a point where the total angle is different from $2 \pi$. The cone angle is the corresponding angle. In what follows we shall assume that the cone angles lie in the interval $(0,2 \pi)$. It is also quite standard to consider cone angles greater than $2 \pi$; see Veech [65] for example. A neighbourhood of a cone singularity with cone angle $\theta_{0} \in(0,2 \pi)$ may be modelled by taking the sector $S_{\alpha}=\left\{z=r e^{i \theta} \in \mathbb{C}: 0 \leq \theta \leq \theta_{0}\right\}$ with the edges identified by the map $r \sim r e^{i \theta_{0}}$ for each $r>0$. A flat cone metric on the sphere is a metric that is locally modelled on the Euclidean metric of $\mathbb{R}^{2}$ except for finitely many points at which there is a cone singularity. A simple example is a cube, which is has eight cone singularities each with cone angle $3 \pi / 2$. Other Euclidean polyhedra correspond to flat cone metrics in the obvious way. We define the curvature at a cone singularity of angle $\theta$ to be $\alpha=2 \pi-\theta$. Thus, away from cone singularities, the curvature is zero. It is easy to see that for any flat cone metric on the sphere the sum of the curvatures at all cone points is equal to $4 \pi$. (So for our example of the cube, there are eight cone singularities each with curvature $\pi / 2$.) Therefore, for any flat cone metric 
on the sphere with $N$ cone singularities with cone angles in $(0,2 \pi)$, the curvatures $\alpha_{1}, \ldots, \alpha_{N}$ satisfy:

$$
\sum_{j=1}^{N} \alpha_{j}=4 \pi, \quad 0<\alpha_{j}<2 \pi \quad \text { for } j=1, \ldots, N .
$$

Comparing equations (3.1) and (3.2) we see that if $\alpha_{1}, \ldots, \alpha_{N}$ are the curvatures at the singularities of a cone metric on the sphere then $\left(\alpha_{1} / 2 \pi, \ldots, \alpha_{N} / 2 \pi\right)$ is a ball $N$-tuple and conversely, given a ball $N$-tuple $\left(\mu_{1}, \ldots, \mu_{N}\right)$ then there exists a flat cone metric on the sphere with curvatures $2 \pi \mu_{1}, \ldots, 2 \pi \mu_{N}$.

Thurston's idea is to consider the space of all flat cone metrics on the sphere with $N$ cone singularities with prescribed curvatures. Allowing the locations of the singularities to vary is equivalent to the way we allowed the points $z_{1}, \ldots, z_{N}$ to vary on the sphere when constructing hypergeometric functions. By cutting along a path joining the cone points, one may unfold a flat cone metric on the sphere to obtain a Euclidean polygon with certain side identifications. Different ways of doing this are described in $[\mathbf{6 3}],[\mathbf{6 6}],[\mathbf{4 7}]$ and $[\mathbf{8}]$. The internal angles of such a polygon are determined by the cone angles, but the side lengths may vary. However paired sides must have the same length. Such a polygon may be described by $N-2$ complex parameters, for example the vectors along the sides. It is not hard to show that the area of this polygon gives a Hermitian form of signature $(1, N-3)$ in these variables. We are only interested in these polygons up to Euclidean similarity. Since a similarity scales all the side vectors, the resulting parameter space may be identified with $\mathbb{C} \mathbb{P}^{N-3}$. By cutting the sphere along different paths between the cone points, we can obtain different polygons from the same cone metric. We can pass from one of these polygons to another by a sequence of Euclidean cut and paste operations. These polygons are related by projective linear transformations in $\operatorname{PGL}(N-2, \mathbb{C})$. Since these transformations preserve the area of the polygon, in fact the projective linear transformations lie in $\mathrm{PU}(1, N-3)$. This is just a new way of viewing the monodromy action of $\pi_{1}(\widetilde{Q})$ described in the previous sections. This idea has been extended to Euclidean cone metrics on other surfaces by Veech, see Theorem 0.9 and Section 14 of [65].

One of Thurston's main results in [63] is a geometric interpretation and refinement of Mostow's result, Theorem 3.2:

Theorem 3.3 (Theorem 0.2 of Thurston [63]). Let $\alpha_{1}, \ldots, \alpha_{N}$ with $N>3$ be a collection of real numbers in the interval $(0,2 \pi)$ whose sum is $4 \pi$. Then the set of Euclidean cone metrics on the sphere with cone points of curvature $\alpha_{j}$ and total area 1 forms a complex hyperbolic manifold whose metric completion is a complex hyperbolic cone manifold $M$ of finite volume. This cone manifold is an orbifold if and only if any pair $\alpha_{j}, \alpha_{k}$ whose sum is less than $2 \pi$ satisfies either

(i) $\left(2 \pi-\alpha_{j}-\alpha_{k}\right)$ divides $2 \pi$, or

(ii) $\alpha_{j}=\alpha_{k}$ and $\pi-\alpha_{j}$ divides $2 \pi$.

We point out that the condition that $M$ is an orbifold is stronger than requiring that the holonomy of $M$ is discrete. In [44] Mostow gave ten ball $N$-tuples which fail the condition $\Sigma$ INT but for which he was unable to prove that the holonomy group was indiscrete. Mostow showed that some of these holonomy groups were arithmetic and hence discrete and Sauter [57] showed that the remaining ones are discrete. 
Motivated by Theorem 3.3 we make the following definition.

Definition 3.4. Let $\alpha_{1}, \ldots, \alpha_{N}$ be curvatures satisfying (3.2). Then the $\alpha_{j}$ are said to satisfy the orbifold condition if any pair $\alpha_{j}, \alpha_{k}$ whose sum is less than $2 \pi$ satisfies either

(i) $\left(2 \pi-\alpha_{j}-\alpha_{k}\right)$ divides $2 \pi$, or

(ii) $\alpha_{j}=\alpha_{k}$ and $\pi-\alpha_{j}$ divides $2 \pi$.

LEMma 3.5. Mostow's condition $\Sigma I N T$ is equivalent to Thurston's orbifold condition.

ProOf. As we have indicated above, we may pass between the ball $N$-tuple $\left(\mu_{1}, \ldots, \mu_{N}\right)$ and the curvatures $\alpha_{1}, \ldots, \alpha_{N}$ by writing $\alpha_{j}=2 \pi \mu_{j}$. In what follows we use the $\mu_{j}$ to avoid having to deal with factors of $2 \pi$. We assume that we are given a ball $N$-tuple satisfying (3.1).

We may restate the orbifold condition as follows. For any pair $\mu_{j}, \mu_{k}$ with $\mu_{j}+\mu_{k}<1$ then either

(i) $1-\mu_{j}-\mu_{k}=1 / n_{j k}$ where $n_{j k} \in \mathbb{Z}$, or

(ii) $\mu_{j}=\mu_{k}$ and $1 / 2-\mu_{j}=1 / m_{j k}$ where $m_{j k} \in \mathbb{Z}$.

It is clear that $\Sigma$ INT implies the orbifold condition. We claim the converse is also true. The main difference between the two criteria concerns the pairs $\mu_{j}, \mu_{k}$ where (ii) is satisfied but not (i). In $\Sigma$ INT all such $\mu_{j}$ take the same value, whereas in the orbifold condition they could take any number of values.

Suppose that we can find a ball $N$-tuple satisfying the orbifold condition but not $\Sigma$ INT. Then (relabelling the indices if necessary) we can find $\mu_{1}=\mu_{2}$ satisfying condition (ii) but not condition (i); and we can find $\mu_{3}=\mu_{4}$ also satisfying (ii) but not (i) and also with $\mu_{1} \neq \mu_{3}$. In other words

$$
1-\mu_{1}-\mu_{2}=2\left(1 / 2-\mu_{1}\right)=2 / m_{12}, \quad 1-\mu_{3}-\mu_{4}=2\left(1 / 2-\mu_{3}\right)=2 / m_{34}
$$

where $m_{12}$ and $m_{34}$ are odd integers. Note that if either of these integers is even then the corresponding pair of $\mu_{j}$ satisfy (i). This means that

$$
\mu_{1}=\mu_{2}=1 / 2-1 / m_{12}, \quad \mu_{3}=\mu_{4}=1 / 2-1 / m_{34} .
$$

Now consider the pair $\mu_{1}$ and $\mu_{3}$. We have $\mu_{1}+\mu_{3}=1-1 / m_{12}-1 / m_{34}<1$ and $\mu_{1} \neq \mu_{3}$. Therefore they must satisfy (i). In other words

$$
1-\mu_{1}-\mu_{3}=1 / n_{13}
$$

where $n_{13}$ is an integer. Now

$$
1-\mu_{1}-\mu_{3}=1 / m_{12}+1 / m_{34}=\left(m_{12}+m_{34}\right) / m_{12} m_{34} .
$$

Therefore $n_{13}=m_{12} m_{34} /\left(m_{12}+m_{34}\right)$. Since $m_{12}$ and $m_{34}$ are odd integers, the numerator is odd and the denominator is even. Hence $n_{13}$ cannot be an integer. This contradiction shows that the orbifold condition implies $\Sigma$ INT as required.

Thurston considers what happens when two or more cone points coalesce. He shows that the locus where this happens is a complex linear submanifold of $\mathbb{C P}^{N-3}$. This complex submanifold is a cone singularity of the moduli space. The stratum $S$ where two cone points coalesce is a complex hyperplane and we can read off the cone angle around $S$ from the original cone points on the sphere. The principle is that the total curvature should remain the same before and after the cone points collide. 
Proposition 3.6 (Proposition 3.5 of Thurston [63]). Let $S$ be a stratum where two cone points with curvatures $\alpha_{i}$ and $\alpha_{j}$ collide. Then the cone angle $\gamma(S)$ around $S$ is $\pi-\alpha_{i}$ if $\alpha_{i}=\alpha_{j}$ and $2 \pi-\alpha_{i}-\alpha_{j}$ otherwise.

We can generalise this result to the case where more than two cone points coalesce. If $j+1$ cone points collide then there is an associated stratum $S$ of complex codimension $j$. The real link of $S$ is the space of real lines normal to $S$, and these are naturally grouped into complex lines, which form the complex link of $S$. The complex link is a complex cone manifold one dimension lower than the real link. The real link is a Seifert fibre space over the complex link. The generic fibres are circles of the same length, and we call this length the scalar cone angle of $S$ and denote it by $\gamma(S)$. Notice that when $j=1$ this is just the cone angle. We define the complex link fraction to be the ratio of the volume of the complex link to the volume of $\mathbb{C P}^{j-1}$. This is the order of the stabiliser of $S$ in $\mathrm{PU}(1, N-3)$. Thurston shows how these two quantities may be calculated.

Proposition 3.7 (Proposition 3.6 of Thurston [63]). Let $S$ be a stratum of complex codimension $j$ where $j+1$ cone points with curvature $\alpha_{1}, \ldots, \alpha_{j}$ collide. Let $m$ be the order of the subgroup of the symmetric group $S_{j}$ that preserves these numbers. Then

(i) the scalar cone angle is $\gamma(S)=2 \pi-\sum_{i=1}^{j} \alpha_{i}$;

(ii) the complex link fraction is $(\gamma(S) / 2 \pi)^{j-1} / m$.

Let $S$ and $S^{\prime}$ be strata where cone points $\left\{v_{1}, \ldots, v_{j}\right\}$ and $\left\{v_{1}^{\prime}, \ldots, v_{k}^{\prime}\right\}$ collide respectively. Suppose that $\left\{v_{1}, \ldots, v_{j}\right\} \cap\left\{v_{1}^{\prime}, \ldots, v_{k}^{\prime}\right\}=\emptyset$. If $S$ and $S^{\prime}$ intersect then the holonomy maps around them commute. This means that the corresponding link fraction is the product of the link fractions around $S$ and $S^{\prime}$.

3.3. Commensurability. Mostow found that there were some ball quintuples that did not satisfy $\Sigma$ INT but yet appeared to correspond to discrete groups. While investigating these groups, Sauter discovered some commensurability theorems [57]. Namely, for each of these exceptional quintuples he found a quintuple satisfying $\Sigma I N T$ so that the associated groups were commensurable. In fact in each case either the groups were isomorphic or else one was isomorphic to a finite index subgroup of the other. Subsequently, Deligne and Mostow discovered further commensurability theorems and this is the main theme of their book [12]. In this paper we will show that all the groups with three fold symmetry are commensurable to one whose fundamental domain is well understood. We shall conclude this section by showing that the same is true for almost all of the quintuples without three fold symmetry.

We begin with Deligne and Mostow's main commensurability theorem. The geometry behind this and the associated calculations are discussed in Chapter 10 of $[\mathbf{1 2}]$.

THEOREM 3.8 (Theorem 10.6 of $[\mathbf{1 2}]$ ). Let $a$ and $b$ be rational numbers with $0<a<1,0<b<1$ and $1 / 2<a+b<1$. Consider the following ball quintuples:

$$
\begin{aligned}
\mu & =(a, a, b, b, 2-2 a-2 b), \\
\nu & =(1-b, 1-a, a+b-1 / 2, a+b-1 / 2,1-a-b) .
\end{aligned}
$$

Let $\Sigma=\mathbb{Z}_{2} \times \mathbb{Z}_{2}$ be the symmetry group of $\mu$ and $\mathrm{T}=\mathbb{Z}_{2}$ be the symmetry group of $\nu$. Then the resulting groups $\Gamma_{\mu \Sigma}$ and $\Gamma_{\nu \mathrm{T}}$ are isomorphic. 
We can use this theorem to give the following result, which is due to Deligne and Mostow, see Corollary 10.18 of [12]. Our formulation is closer to the one given by Sauter on page 354 of Sauter [57], and which generalises Theorem 6.2 of [57]. We have been deliberately ambiguous about the symmetry groups in operation here, see (10.15.1) of [12] for the precise relationship. We give a more precise reformulation of part of this result in Proposition 4.10 below.

Corollary 3.9 (Corollary 10.18 of $[\mathbf{1 2}]$ ). The groups $\Gamma_{\mu^{(j)}}$ with

$$
\begin{aligned}
\mu^{(1)} & =\left(\frac{1}{2}-\frac{1}{p}, \frac{1}{2}-\frac{1}{p}, \frac{1}{2}-\frac{1}{p}, \frac{1}{2}-\frac{1}{p}, \frac{4}{p}\right), \\
\mu^{(2)} & =\left(\frac{1}{2}+\frac{1}{p}, \frac{1}{2}+\frac{1}{p}, \frac{1}{2}-\frac{2}{p}, \frac{1}{2}-\frac{2}{p}, \frac{2}{p}\right), \\
\mu^{(3)} & =\left(\frac{1}{2}-\frac{1}{p}, \frac{1}{2}-\frac{1}{p}, \frac{1}{2}-\frac{1}{p}, \frac{1}{p}, \frac{1}{2}+\frac{2}{p}\right)
\end{aligned}
$$

are commensurable. They correspond to lattices when $p \in\{5,6,7,8,9,10,12,18\}$.

Proof. First putting $a=b=1 / 2-1 / p$ in Theorem 3.8 gives $\mu^{(1)}$ and $\mu^{(2)}$. Then putting $a=1 / 2+1 / p$ and $b=1 / 2-2 / p$ in Theorem 3.8 gives $\mu^{(2)}$ and $\mu^{(3)}$. The quintuples $\mu^{(1)}$ and $\mu^{(3)}$ satisfy the orbifold condition whenever $p \in\{5,6,7,8,9,10,12,18\}$. However, $\mu^{(2)}$ only satisfies the orbifold condition when $p \in\{6,8,10,12,18\}$.

COROLlary 3.10. The groups $\Gamma_{\mu^{(j)}}$ with

$$
\begin{aligned}
\mu^{(1)} & =\left(\frac{1}{2}-\frac{1}{k}, \frac{1}{2}-\frac{1}{k}, \frac{1}{4}+\frac{1}{k}, \frac{1}{4}+\frac{1}{k}, \frac{1}{2}\right), \\
\mu^{(2)} & =\left(\frac{1}{4}, \frac{1}{4}, \frac{1}{4}, \frac{3}{4}-\frac{1}{k}, \frac{1}{2}+\frac{1}{k}\right)
\end{aligned}
$$

are commensurable. They correspond to lattices when $k \in\{3,4,5,6,8\}$.

Proof. We put $a=1 / 2-1 / k$ and $b=1 / 4+1 / k$ in Theorem 3.8. Both $\mu^{(1)}$ and $\mu^{(2)}$ satisfy the orbifold condition when $k \in\{3,4,5,6,8\}$.

There is another similar theorem due to Sauter, Theorem 6.1 of [57] (see also Theorem 11.22 of $[\mathbf{1 2}]$ ). Once again, we shall reformulate this result in Proposition 4.9 below.

Theorem 3.11 (Theorem 6.1 of $[\mathbf{5 7}]$ ). The groups $\Gamma_{\mu^{(j)}}$ with

$$
\begin{aligned}
\mu^{(1)} & =\left(\frac{1}{2}-\frac{1}{m}, \frac{1}{2}-\frac{1}{m}, \frac{1}{2}-\frac{1}{m}, \frac{1}{6}+\frac{1}{m}, \frac{1}{3}+\frac{2}{m}\right), \\
\mu^{(2)} & =\left(\frac{1}{6}, \frac{1}{6}, \frac{1}{6}, \frac{5}{6}-\frac{1}{m}, \frac{2}{3}+\frac{1}{m}\right)
\end{aligned}
$$

are commensurable. They correspond to lattices when

$$
m \in\{4,5,6,7,8,9,10,12,15,18,24,42, \infty,-30,-12\} .
$$


Note that the quintuple $\mu^{(2)}$ in Theorem 3.11 satisfies the orbifold condition for all the given values of $m$. On the other hand, $\mu^{(1)}$ satisfies the orbifold condition only when 3 divides $6 m /(m-6)$, that is when $m \in\{7,8,9,10,12,18, \infty\}$. (In the case $m=\infty$ we regard $6 m /(m-6)=6 /(1-6 / m)=6$.)

Finally, we list those ball quintuples that do not have three fold symmetry and which are associated to lattices. This list may be found in Section 7 of Sauter [57]. For simplicity, we give the groups with three fold symmetry in terms of the pair $(p, k)$ as described in the next section. There are five groups that are not related to a group with three fold symmetry. Of these five groups two pairs are related by Theorem 3.8 and we have indicated these by (a) and (b). The fifth does not seem to be related to any other group. We have labelled it (c). The last column indicates which ones are arithmetic A or non-arithmetic N.

\begin{tabular}{|rrrrr|l|l|l|l|}
\hline$\mu_{1}$ & $\mu_{2}$ & $\mu_{3}$ & $\mu_{4}$ & $\mu_{5}$ & $3.9(p, k)$ & $3.10(p, k)$ & 3.8 & \\
\hline $1 / 10$ & $1 / 10$ & $7 / 10$ & $7 / 10$ & $2 / 5$ & $(5,2)$ & & & $A$ \\
$1 / 6$ & $1 / 6$ & $2 / 3$ & $2 / 3$ & $1 / 3$ & $(6,2)$ & & & $A$ \\
$3 / 14$ & $3 / 14$ & $9 / 14$ & $9 / 14$ & $2 / 7$ & $(7,2)$ & & & $A$ \\
$5 / 18$ & $5 / 18$ & $11 / 18$ & $11 / 18$ & $2 / 9$ & $(9,2)$ & & & $N$ \\
$3 / 10$ & $3 / 10$ & $3 / 5$ & $3 / 5$ & $1 / 5$ & $(10,2)$ & & & $A$ \\
$1 / 3$ & $1 / 3$ & $7 / 12$ & $7 / 12$ & $1 / 6$ & $(12,2)$ & & & $A$ \\
$7 / 18$ & $7 / 18$ & $5 / 9$ & $5 / 9$ & $1 / 9$ & $(18,2)$ & & & $A$ \\
$1 / 6$ & $1 / 6$ & $7 / 12$ & $7 / 12$ & $1 / 2$ & & $(4,3)$ & & $A$ \\
$3 / 10$ & $3 / 10$ & $9 / 20$ & $9 / 20$ & $1 / 2$ & & $(4,5)$ & & $N$ \\
$1 / 3$ & $1 / 3$ & $5 / 12$ & $5 / 12$ & $1 / 2$ & & $(4,6)$ & & $N$ \\
$1 / 4$ & $1 / 4$ & $5 / 12$ & $5 / 12$ & $2 / 3$ & & & $(a)$ & $A$ \\
$1 / 6$ & $1 / 6$ & $1 / 2$ & $1 / 2$ & $2 / 3$ & & & $(b)$ & $A$ \\
\hline $5 / 12$ & $5 / 12$ & $1 / 12$ & $1 / 4$ & $5 / 6$ & & & $(3,12)$ & $A$ \\
$1 / 3$ & $1 / 3$ & $1 / 6$ & $1 / 2$ & $2 / 3$ & & & $(6,6)$ & $A$ \\
$1 / 6$ & $1 / 6$ & $1 / 3$ & $7 / 12$ & $3 / 4$ & & & $(a)$ & $A$ \\
$1 / 6$ & $1 / 6$ & $1 / 3$ & $1 / 2$ & $5 / 6$ & & & $(b)$ & $A$ \\
$1 / 4$ & $1 / 4$ & $5 / 12$ & $1 / 2$ & $7 / 12$ & & & $(c)$ & $N$ \\
\hline
\end{tabular}

\section{Deligne-Mostow groups with three fold symmetry}

In this section we consider Deligne-Mostow groups arising from ball quintuples with $\mu_{1}=\mu_{2}=\mu_{3}$. Equivalently, we consider the modular group associated to cone metrics on the sphere with five cone singularities, three of which have the same angle.

4.1. Ball quintuples and cone metrics with three fold symmetry. Suppose we have a ball quintuple $\mu=\left(\mu_{1}, \mu_{2}, \mu_{3}, \mu_{4}, \mu_{5}\right)$ where $\mu_{1}=\mu_{2}=\mu_{3}$. The curvature at the cone point $v_{j}$ is $\alpha_{j}=2 \pi \mu_{j}$. Using Proposition 3.7 we see that the cone angle around each stratum where two of $v_{1}, v_{2}, v_{3}$ have collided is $\pi-\alpha_{1}$. We define $p$ by requiring that this angle be $2 \pi / p$. Therefore we choose $\mu_{1}=\mu_{2}=\mu_{3}=1 / 2-1 / p$. From the orbifold condition, or equivalently $\Sigma$ INT, we see that $p$ is an integer. Likewise, when $\alpha_{1} \neq \alpha_{4}$ the cone angle around each stratum where one of $v_{1}, v_{2}, v_{3}$ has collided with $v_{4}$ is $2 \pi-\alpha_{1}-\alpha_{4}$. We define this to be $2 \pi / k$ and so $\mu_{4}=1 / 2+1 / p-1 / k$. If $\mu$ satisfies the orbifold condition then $k$ will be an integer. It may be that $\alpha_{1}=\alpha_{4}$. In this case the cone angle around 


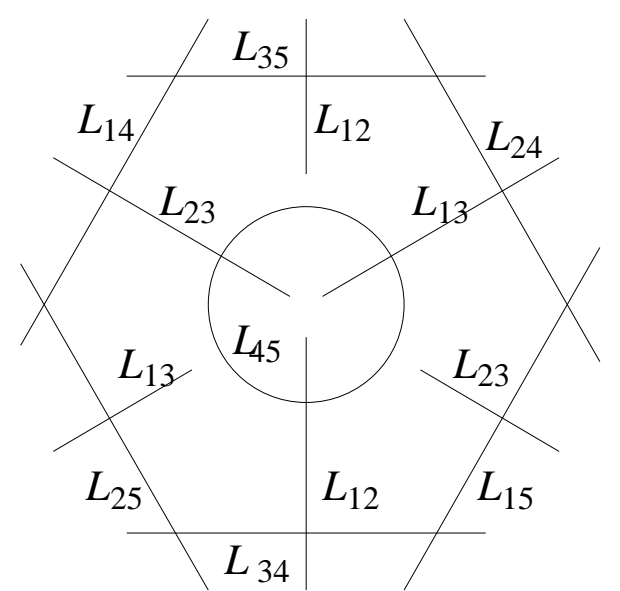

Figure 2. The line arrangement when $l>0$ and $d>0$. The labels refer to the labels of the pair of cone points associated to each stratum.

each of these strata will be $2 \pi / p$. For consistency in our formulae we still write $\mu_{4}=1 / 2+1 / p-1 / k$ and so $k=p / 2$. Note that in this case, even though $k$ may not be an integer, the quintuple $\mu$ still satisfies the orbifold condition. It has the form of $\mu^{(1)}$ in Corollary 3.9.

The condition that the $\mu_{j}$ should sum to 2 means that $\mu_{5}=2 / p+1 / k$. In other words, we have cone singularities $v_{j}$ with curvatures $\alpha_{j}=2 \pi \mu_{j}$ where

$$
\mu_{1}=\mu_{2}=\mu_{3}=\frac{1}{2}-\frac{1}{p}, \quad \mu_{4}=\frac{1}{2}+\frac{1}{p}-\frac{1}{k}, \quad \mu_{5}=\frac{2}{p}+\frac{1}{k} .
$$

The cone angle around each stratum where one of $v_{1}, v_{2}, v_{3}$ has collided with $v_{5}$ is $2 \pi-\alpha_{1}-\alpha_{5}$. Write this as $2 \pi / l$ and so $\mu_{5}=1 / 2+1 / p-1 / l$. In other words, $1 / l=1 / 2-1 / p-1 / k$. If $l>0$ and $\mu$ satisfies the orbifold condition then $l$ will be an integer. The cone angle around the stratum where $v_{4}$ and $v_{5}$ have collided is $2 \pi-\alpha_{4}-\alpha_{5}$. Write this as $2 \pi / d$ and so $1 / d=1 / 2-3 / p$. If $d>0$ and $\mu$ satisfies the orbifold condition then $d$ will be an integer.

When two cone points $v_{i}$ and $v_{j}$ collide the associated stratum is a complex line, which we call $L_{i j}$. When three cone points $v_{i}, v_{j}, v_{k}$ collide the associated stratum is a point, which we denote by $z_{i j k}$. In the following table we use Proposition 3.7 to list the strata corresponding to where various cone points have collided. There are other strata corresponding to permuting $v_{1}, v_{2}$ and $v_{3}$.

\begin{tabular}{|l|l|l|l|}
\hline Stratum & Cone points & (Scalar) cone angle & Link fraction \\
\hline$L_{12}$ & $v_{1}, v_{2}$ & $\pi-\alpha_{1}=2 \pi / p$ & $1 / p$ \\
$L_{14}$ & $v_{1}, v_{4}$ & $2 \pi-\alpha_{1}-\alpha_{4}=2 \pi / k$ & $1 / k$ \\
$L_{15}$ & $v_{1}, v_{5}$ & $2 \pi-\alpha_{1}-\alpha_{5}=2 \pi / l,(l>0)$ & $1 / l$ \\
$L_{45}$ & $v_{4}, v_{5}$ & $2 \pi-\alpha_{4}-\alpha_{5}=2 \pi / d,(d>0)$ & $1 / d$ \\
$z_{134}$ & $v_{1}, v_{3}, v_{4}$ & $2 \pi-2 \alpha_{1}-\alpha_{4}=-2 \pi / l,(l<0)$ & $1 / 2 l^{2}$ \\
$z_{123}$ & $v_{1}, v_{2}, v_{3}$ & $2 \pi-3 \alpha_{1}=-2 \pi / d,(d<0)$ & $1 / 6 d^{2}$ \\
\hline
\end{tabular}

Note that there are no strata corresponding to $z_{125}$ and $z_{145}$ as the scalar cone angles would be $-2 \pi / k$ and $-4 \pi / p$ respectively. When $\{i, j\} \cap\{k, l\}=\emptyset$ the holonomy 


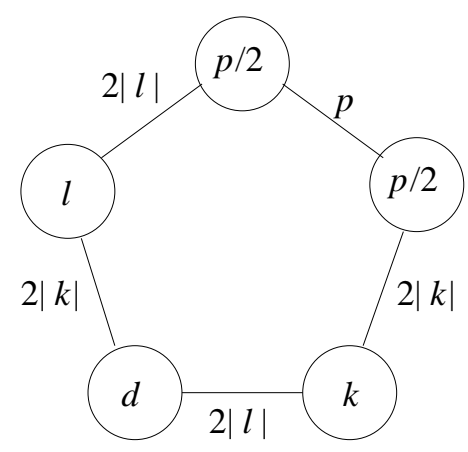

Figure 3. Yoshida's graph for the quintuple associated to $p$ and $k$. The edges are drawn using different styles of lines as indicated in Figure 1.

map around $L_{i j}$ commutes with the holonomy map around $L_{k l}$. In this case we let $z_{i j, k l}$ denote $L_{i j} \cap L_{k l}$.

Using this table, we see that when $l>0$ and $d>0$ there are no strata associated to the collisions of three cone points. In this case, there are ten complex lines, each associated to the collision of two cone points. Each of these complex lines intersects three of the others. Each intersection consists of two pairs of distinct cone points and so the strata intersect orthogonally. We have indicated this in Figure 2, which should be compared to the figure on page 78 of Deligne and Mostow [12], Figure 5.3 of Sauter [57] or Figure 1 of Toledo [64]. The associated Yoshida graph is given in Figure 3. The edge joining the two vertices labelled $p / 2$ is solid, bold or dashed when $1 / d<0,=0,>0$ respectively. The edge joining the vertices labelled $p / 2$ and $k$ is solid, bold or dashed when $1 / l<0,=0,>0$. The edge joining the vertices labelled $p / 2$ and $l$ is solid, bold or dashed when $1 / k<0,=0,>0$. Finally the edges joining the vertex labelled $d$ are solid, bold or dashed when $1 / p<0,=0,>0$.

4.2. Monodromy groups and equilateral triangle groups. Consider the map $R_{1}$, which is the holonomy around $L_{23}$. This is a complex reflection fixing $L_{23}$ with angle $2 \pi / p$. Similarly, $R_{2}$ and $A_{1}$ are the holonomies around $L_{13}$ and $L_{14}$. These are complex reflections with angles $2 \pi / p$ and $2 \pi / k$.

Thinking of the cone points $v_{1}, \ldots, v_{5}$ as marked points on the sphere, we can think of $R_{1}, R_{2}, A_{1}$ as elements of the mapping class group of the five punctured sphere. There is a well known relation between the mapping class group and the braid group; see Birman's book [7]. Mostow uses this connection to write $R_{1}, R_{2}$ and $A_{1}$ as elements of the braid group; see page 243 of [43] or page 337 of Sauter [57]. A straightforward consequence of this is that $R_{1}$ and $A_{1}$ commute and $R_{1}$ and $R_{2}$ satisfy the braid relation. That is

$$
R_{1} A_{1}=A_{1} R_{1}, \quad R_{1} R_{2} R_{1}=R_{2} R_{1} R_{2} .
$$

In Figure 4 we show how to obtain $J=R_{1} R_{2} A_{1}$ in terms of the braid picture. If the points $v_{4}$ and $v_{5}$ are placed at the north and south poles of a sphere and the points $v_{1}, v_{2}$ and $v_{3}$ are equally spaced around the equator, then $J$ is a rotation of the sphere through an angle $2 \pi / 3$ that fixes the poles; see Figure 15 of $[43]$. The map $J^{3}$ has the effect of rotating once about $v_{5}$. This is clearly isotopic to the identity. Thus $J$ has order 3 . In Figure 4 we have drawn the three cone points $v_{1}$, 


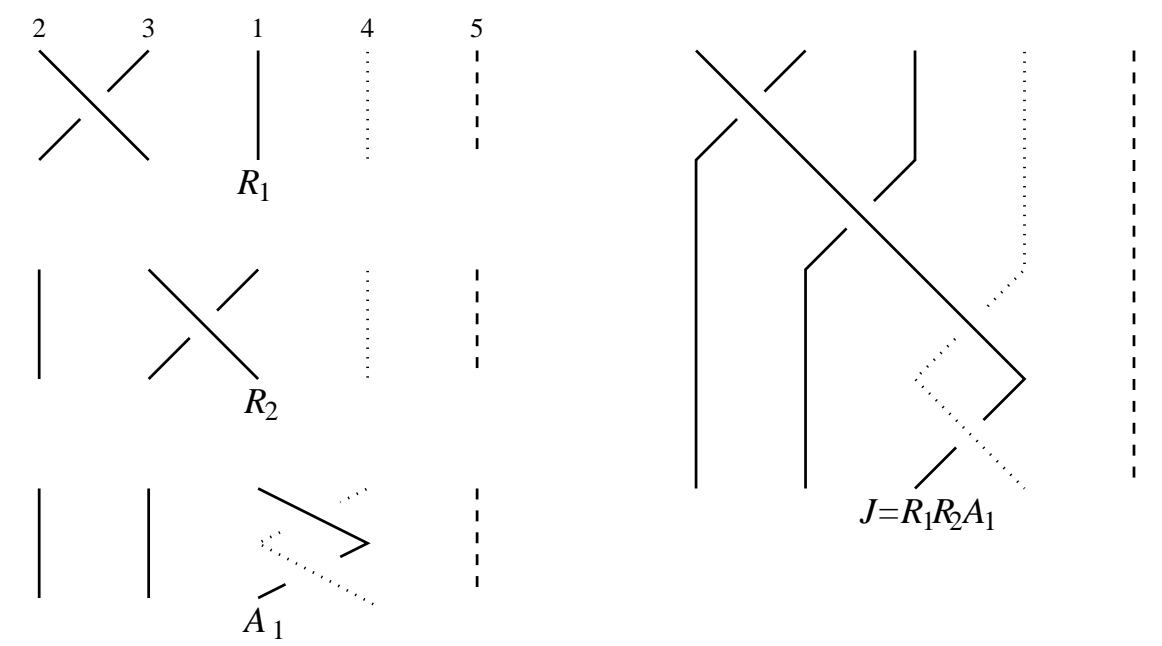

Figure 4. The maps $R_{1}, R_{2}, A_{1}$ and $J$ as braids.

$v_{2}, v_{3}$ with curvature $\alpha_{1}$ as solid lines, the cone point $v_{4}$ as a dotted line and the cone point $v_{5}$ as a dashed line. Each automorphism of the cone structure must map $v_{4}$ and $v_{5}$ to themselves but is allowed to permute $v_{1}, v_{2}$ and $v_{3}$.

As indicated above the map $J=R_{1} R_{2} A_{1}$ has order 3 . Moreover, using the identities (4.1) we have

$$
J R_{1} J^{-1}=R_{1} R_{2} A_{1} R_{1} A_{1}^{-1} R_{2}^{-1} R_{1}^{-1}=R_{2} .
$$

Define $R_{3}=J R_{2} J^{-1}=J^{-1} R_{1} J$. Then $R_{1}, R_{2}$ and $R_{3}$ are each complex reflections in a complex lines. These complex lines form a triangle in complex hyperbolic space and we say that $\left\langle R_{1}, R_{2}, R_{3}\right\rangle$ is a complex hyperbolic triangle group. Because this triangle has a symmetry $J$ of order 3 we call it equilateral. To summarise:

$$
J^{3}=I, \quad R_{2}=J R_{1} J^{-1}, \quad R_{3}=J R_{2} J^{-1}=J^{-1} R_{1} J .
$$

We should point out that the symmetry $J$ does not correspond to the obvious three fold rotational symmetry of the line arrangement diagrams. That is, if $L_{23}$ is the mirror of $R_{1}$ and $L_{31}$ is the mirror of $R_{2}$ then $L_{12}$ is the mirror of $R_{2}^{-1} R_{1} R_{2}=R_{1} R_{2} R_{1}^{-1}$ and not of $R_{3}$.

From the definition of $J$ we have $A_{1}=R_{2}^{-1} R_{1}^{-1} J$. Following Mostow, [43] equation (5.3), and Sauter, [57] equation (2.6), for indices $j=1,2,3$ taken mod 3 , we define

$$
A_{j}=R_{j+1}^{-1} R_{j}^{-1} J, \quad A_{j}^{\prime}=R_{j-1}^{-1} R_{j}^{-1} J^{-1}=J^{-1} R_{j}^{-1} R_{j+1}^{-1} .
$$

These maps enable us to write down the holonomy around each of the strata $L_{i j}$ and $z_{i j k}$ described in the previous section. In the following table we summarise this data. In each case we write down the stabiliser and its order. In each case the order of the stabiliser is the reciprocal of the link fraction. The stabilisers of $L_{j k}$ are all cyclic. We discuss the group structure of the stabilisers of $z_{j k}$ in Propositions 4.4, 
4.5 and 4.6 below.

\begin{tabular}{|l|l|l|l|}
\hline Stratum & Cone points & Stabiliser & Order \\
\hline$L_{23}$ & $v_{2}, v_{3}$ & $\left\langle R_{1}\right\rangle$ & $p$ \\
$L_{13}$ & $v_{1}, v_{3}$ & $\left\langle R_{2}\right\rangle$ & $p$ \\
$L_{12}$ & $v_{1}, v_{2}$ & $\left\langle R_{2}^{-1} R_{1} R_{2}\right\rangle$ & $p$ \\
$L_{14}$ & $v_{1}, v_{4}$ & $\left\langle A_{1}\right\rangle$ & $k$ \\
$L_{24}$ & $v_{2}, v_{4}$ & $\left\langle A_{2}\right\rangle$ & $k$ \\
$L_{34}$ & $v_{3}, v_{4}$ & $\left\langle R_{2}^{-1} A_{1} R_{2}\right\rangle$ & $k$ \\
$L_{15}$ & $v_{1}, v_{5}$ & $\left\langle A_{1}^{\prime}\right\rangle$ & $l(l>0)$ \\
$L_{45}$ & $v_{4}, v_{5}$ & $\left\langle\left(R_{1} R_{2}\right)^{3}\right\rangle$ & $d(d>0)$ \\
$z_{134}$ & $v_{1}, v_{3}, v_{4}$ & $\left\langle R_{2}, A_{1}\right\rangle$ & $2 l^{2}(l<0)$ \\
$z_{123}$ & $v_{1}, v_{2}, v_{3}$ & $\left\langle R_{1}, R_{2}\right\rangle$ & $6 d^{2}(d<0)$ \\
\hline
\end{tabular}

Stabilisers of strata corresponding to distinct sets of cone points commute. Thus, some obvious consequences of the above table are, for $j \neq k$,

$$
R_{j} A_{j}=A_{j} R_{j}, \quad R_{j} A_{j}^{\prime}=A_{j}^{\prime} R_{j}, \quad A_{j} A_{k}^{\prime}=A_{k}^{\prime} A_{j}, \quad R_{j}\left(R_{j} R_{k}\right)^{3}=\left(R_{j} R_{k}\right)^{3} R_{j} .
$$

Therefore, when $\{i, j\} \cap\{k, l\}=\emptyset$, the stabilisers of the stratum $z_{i j, k l}=L_{i j} \cap L_{k l}$ is simply the direct product of the stabilisers of $L_{i j}$ and $L_{k l}$.

\begin{tabular}{|l|l|l|l|}
\hline Stratum & Strata & Stabiliser & Order \\
\hline$z_{23,14}$ & $L_{23}, L_{14}$ & $\left\langle R_{1}, A_{1}\right\rangle$ & $k p$ \\
$z_{23,15}$ & $L_{23}, L_{15}$ & $\left\langle R_{1}, A_{1}^{\prime}\right\rangle$ & $l p(l>0)$ \\
$z_{24,15}$ & $L_{14}, L_{25}$ & $\left\langle A_{2}, A_{1}^{\prime}\right\rangle$ & $k l(l>0)$ \\
$z_{23,45}$ & $L_{23}, L_{45}$ & $\left\langle R_{1},\left(R_{1} R_{2}\right)^{3}\right\rangle$ & $d p(d>0)$ \\
\hline
\end{tabular}

We now briefly discuss the special case where $k=p / 2$; see Theorems 5.6 and 6.2 of Sauter [57]. In this case

$$
\mu_{1}=\mu_{2}=\mu_{3}=\mu_{4}=\frac{1}{2}-\frac{1}{p}, \quad \mu_{5}=\frac{4}{p} .
$$

Because four of the $\mu_{j}$ are the same, we have the symmetry group $\Sigma=S_{4}$ acting. This means that for $j=1,2,3$ the holonomy around $L_{j 4}$ is a complex reflection with angle $2 \pi / p$. This is a square root of $A_{j}$ which we denote by $R_{j}^{\prime}$. From the braid group picture, it is clear that, for $j \neq k$, we have

$$
R^{\prime 2}=A_{j}, \quad R_{j}^{\prime} R_{j}=R_{j} R_{j}^{\prime}, \quad R_{j} R_{k}^{\prime} R_{j}=R_{k}^{\prime} R_{j} R_{k}^{\prime}, \quad R_{j}^{\prime} R_{k}^{\prime} R_{j}^{\prime}=R_{k}^{\prime} R_{j}^{\prime} R_{k}^{\prime} .
$$

As discussed above, complex reflections $R_{1}, R_{2}, R_{3}$ with a symmetry $J$ satisfying (4.2) form an equilateral complex hyperbolic triangle group. In [48] for the case when the $R_{j}$ have order two, and in [50] for the general case, Parker and Paupert have shown that, up to conjugation, equilateral complex triangle groups may be parametrised by $\tau=\operatorname{tr}\left(R_{1} J\right)$. Using a normalisation similar to that of Mostow [41] they then show that we may take the Hermitian form $H$ to be

$$
H=\left[\begin{array}{ccc}
2 \sin (\pi / p) & -i e^{-\pi i / 3 p} \tau & i e^{\pi i / 3 p} \bar{\tau} \\
i e^{\pi i / 3 p} \bar{\tau} & 2 \sin (\pi / p) & -i e^{-\pi i / 3 p} \tau \\
-i e^{-\pi i / 3 p} \tau & i e^{\pi i / 3 p} \bar{\tau} & 2 \sin (\pi / p)
\end{array}\right]
$$


This leads to the following matrices in $\mathrm{SU}(H)$ for $R_{1}, R_{2}, R_{3}$ and $J$ :

$$
\begin{aligned}
& R_{1}=\left[\begin{array}{ccc}
e^{4 \pi i / 3 p} & \tau & -e^{2 \pi i / 3 p} \bar{\tau} \\
0 & e^{-2 \pi i / 3 p} & 0 \\
0 & 0 & e^{-2 \pi i / 3 p}
\end{array}\right], \quad R_{2}=\left[\begin{array}{ccc}
e^{-2 \pi i / 3 p} & 0 & 0 \\
-e^{2 \pi i / 3 p} \bar{\tau} & e^{4 \pi i / 3 p} & \tau \\
0 & 0 & e^{-2 \pi i / 3 p}
\end{array}\right], \\
& R_{3}=\left[\begin{array}{ccc}
e^{-2 \pi i / 3 p} & 0 & 0 \\
0 & e^{-2 \pi i / 3 p} & 0 \\
\tau & -e^{2 \pi i / 3 p} \bar{\tau} & e^{4 \pi i / 3 p}
\end{array}\right], \quad J=\left[\begin{array}{ccc}
0 & 0 & 1 \\
1 & 0 & 0 \\
0 & 1 & 0
\end{array}\right] \text {. }
\end{aligned}
$$

The form $H$ has signature $(2,1)$ provided

$$
\operatorname{det}(H)=8 \sin ^{3}(\pi / p)-6 \sin (\pi / p)|\tau|^{2}+2 \operatorname{Re}\left(i e^{-i \pi / p} \tau^{3}\right)<0 .
$$

Before continuing, we remark that this construction is consistent when $p=\infty$. Here $\pi / p=0$ and so $H$ and $R_{1}$ become

$$
H=\left[\begin{array}{ccc}
0 & -i \tau & i \bar{\tau} \\
i \bar{\tau} & 0 & -i \tau \\
-i \tau & i \bar{\tau} & 0
\end{array}\right], \quad R_{1}=\left[\begin{array}{ccc}
1 & \tau & -\bar{\tau} \\
0 & 1 & 0 \\
0 & 0 & 1
\end{array}\right]
$$

In this case $R_{1}$ is unipotent with a two dimensional eigenspace. This means that it is conjugate to a vertical Heisenberg translation (see Section 4.2 of Goldman [20]). The form $H$ has signature $(2,1)$ provided $\operatorname{det}(H)=2 \operatorname{Re}\left(i \tau^{3}\right)<0$.

Given an equilateral complex hyperbolic triangle group, as in equation (4.3) we define $A_{j}=R_{j+1}^{-1} R_{j}^{-1} J=\left(J R_{j}^{-1} J\right)^{2}$. This is a complex reflection fixing of order $k$. (In the case where $k=\infty$ then $A_{j}$ is conjugate to a vertical Heisenberg translation.) Conjugating the identities (4.1) by powers of $J$ leads to

$$
R_{j} A_{j}=A_{j} R_{j}, \quad R_{j} R_{k} R_{j}=R_{k} R_{j} R_{k} .
$$

We now characterise this condition in terms of $\tau$.

Proposition 4.1. Let $J$ and $R_{j}$ for $j=1,2,3$ be defined by (4.2). Define $A_{j}=R_{j+1}^{-1} R_{j} J=\left(J R_{j}^{-1} J\right)^{2}$ and $\tau=\operatorname{tr}\left(R_{j} J\right)$. The following are equivalent:

(i) $A_{j}$ is a complex reflection or is conjugate to a vertical Heisenberg translation:

(ii) $|\tau|=1$;

(iii) $R_{j}$ commutes with $A_{j}$;

(iv) $R_{j}$ and $R_{k}$ satisfy the braid relation $R_{j} R_{k} R_{j}=R_{k} R_{j} R_{k}$.

ProOF.

- (i) and (ii) are equivalent. Assume that $A_{j}$ either is a complex reflection or is conjugate to a vertical Heisenberg translation. In particular, $A_{j}$ has a repeated eigenvalue. A matrix in $\mathrm{SU}(2,1)$ has a repeated eigenvalue if and only if its trace is a zero of the discrminiant function $f(z)=|z|^{4}-4\left(z^{3}+\bar{z}^{3}\right)+18|z|^{2}-27$; see page 207 of Goldman [20]. Since $\tau=\operatorname{tr}\left(R_{j} J\right)$ and $A_{j}=J^{-1}\left(R_{j} J\right)^{-2} J$, an easy computation shows that

$$
\operatorname{tr}\left(A_{j}\right)=\bar{\tau}^{2}-2 \tau \text {. }
$$

(To see this, one may either multiply matrices in the standard form given in [50] or else examine eigenvalues.) Putting this into the discriminant function and simplifying we see that

$$
f\left(\operatorname{tr}\left(A_{j}\right)\right)=\left(|\tau|^{2}-1\right)^{2}\left(|\tau|^{4}-4\left(\tau^{3}+\bar{\tau}^{3}\right)+18|\tau|^{2}-27\right) .
$$


This vanishes if and only if either $|\tau|=1$ or $f(\tau)=0$. If $|\tau|=1$ we have condition (ii). Therefore, we suppose that $f(\tau)=0$ and so $R_{j} J$ has a repeated eigenvalue. Using the standard form from [50], it is easy to check that the eigenspace of $R_{j} J$ corresponding to its repeated eigenvalue is one dimensional. Hence $R_{j} J$ is parabolic. Therefore $A_{j}=J^{-1}\left(R_{j} J\right)^{-2} J$ is also parabolic and, by hypothesis, is conjugate to a vertical Heisenberg translation. In particular, $\operatorname{tr}\left(A_{j}\right)=3$ and so $\tau=-1$ or $\tau=3$. Substituting in (4.6) we see that when $\tau=3$ then $H$ does not have signature $(2,1)$. Thus $\tau=-1$. Hence, if $A_{j}$ is a complex reflection or a Heisenberg translation then $|\tau|=1$.

Conversely, when $|\tau|=1$, the formula above implies $f\left(\operatorname{tr}\left(A_{j}\right)\right)=0$. We need to show that $A_{j}$ is neither a screw parabolic map nor conjugate to a non-vertical Heisenberg translation. This follows by substituting for $|\tau|$ in the normal form of [50] given above.

- (ii) and (iii) are equivalent. A similar calculation shows that

$$
\operatorname{tr}\left[R_{j}, A_{j}\right]=3+\left(|\tau|^{2}-1\right)^{2}\left(|\tau|^{2}-2 \sin ^{2}(\pi / p)\right) .
$$

Therefore, if $\operatorname{tr}\left[R_{j}, A_{j}\right]=3$ then either $|\tau|=1$ or $|\tau|=2 \sin (\pi / p)$. Using the normal form of [50] given above, we see that if $|\tau|=1$ then $\left[R_{j}, A_{j}\right]$ is the identity and if $|\tau|=2 \sin (\pi / p)$ then $\left[R_{j}, A_{j}\right]$ is parabolic.

- (iii) and (iv) are equivalent. This will follow from the following identity:

$$
\begin{aligned}
A_{j} R_{j}^{-1} A_{j}^{-1} R_{j} & =R_{j+1}^{-1} R_{j}^{-1} J R_{j}^{-1} J^{-1} R_{j} R_{j+1} R_{j} \\
& =R_{j+1}^{-1} R_{j}^{-1} R_{j+1}^{-1} R_{j} R_{j+1} R_{j} .
\end{aligned}
$$

Corollary 4.2. Using the notation of Proposition 4.1, if $A_{j}$ is a complex reflection with angle $2 \pi / k$ then $\tau=-e^{-2 \pi i / 3 k}$ and if $A_{j}$ is conjugate to a vertical Heisenberg translation then $\tau=-1$.

Proof. We have already seen that if $A_{j}$ is a complex reflection then $|\tau|=1$ and $\operatorname{tr}\left(A_{j}\right)=\bar{\tau}^{2}-2 \tau$. Hence, we see that the angle of $A_{j}$ is the argument of $-\bar{\tau}^{3}$. This gives the result.

Putting in $\tau=-e^{-2 \pi i / 3 k}$ (including $\tau=-1$ when $k=\infty$ ) in Parker and Paupert's expressions for $R_{1}, R_{2}$ and $R_{3}$, we obtain:

$$
\begin{aligned}
& R_{1}=\left[\begin{array}{ccc}
e^{4 \pi i / 3 p} & -e^{-2 \pi i / 3 k} & e^{2 \pi i / 3 p+2 \pi i / 3 k} \\
0 & e^{-2 \pi i / 3 p} & 0 \\
0 & 0 & e^{-2 \pi i / 3 p}
\end{array}\right], \\
& R_{2}=\left[\begin{array}{ccc}
e^{-2 \pi i / 3 p} & 0 & 0 \\
e^{2 \pi i / 3 p+2 \pi i / 3 k} & e^{4 \pi i / 3 p} & -e^{-2 \pi i / 3 k} \\
0 & 0 & e^{-2 \pi i / 3 p}
\end{array}\right], \\
& R_{3}=\left[\begin{array}{ccc}
e^{-2 \pi i / 3 p} & 0 & 0 \\
0 & e^{-2 \pi i / 3 p} & 0 \\
-e^{-2 \pi i / 3 k} & e^{2 \pi i / 3 p+2 \pi i / 3 k} & e^{4 \pi i / 3 p}
\end{array}\right] .
\end{aligned}
$$


Using $A_{j}=R_{j+1}^{-1} R_{j}^{-1} J$ we obtain explicit formulae for the $A_{j}$.

$$
\begin{aligned}
& A_{1}=\left[\begin{array}{ccc}
e^{-2 \pi i / 3 k} & -e^{2 \pi i / 3 p+2 \pi i / 3 k} & e^{-2 \pi i / 3 p} \\
0 & e^{4 \pi i / 3 k}+e^{-2 \pi i / 3 k} & -e^{-4 \pi i / 3 p+2 \pi i / 3 k} \\
0 & e^{4 \pi i / 3 p} & 0
\end{array}\right], \\
& A_{2}=\left[\begin{array}{ccc}
0 & 0 & e^{4 \pi i / 3 p} \\
e^{-2 \pi i / 3 p} & e^{-2 \pi i / 3 k} & -e^{2 \pi i / 3 p+2 \pi i / 3 k} \\
-e^{-4 \pi i / 3 p+2 \pi i / 3 k} & 0 & e^{4 \pi i / 3 k}+e^{-2 \pi i / 3 k}
\end{array}\right], \\
& A_{3}=\left[\begin{array}{ccc}
e^{4 \pi i / 3 k}+e^{-2 \pi i / 3 k} & -e^{-4 \pi i / 3 p+2 \pi i / 3 k} & 0 \\
e^{4 \pi i / 3 p} & 0 & 0 \\
-e^{2 \pi i / 3 p+2 \pi i / 3 k} & e^{-2 \pi i / 3 p} & e^{-2 \pi i / 3 k}
\end{array}\right] .
\end{aligned}
$$

Lemma 4.3. The Hermitian form $H$ given by $(4.5)$ has signature $(2,1)$ whenever $\mu_{4}, \mu_{5} \in(0,1)$.

Proof. Put $\tau=-e^{-2 \pi i / 3 k}$ in (4.6). In order to show that $H$ has signature $(2,1)$ we must show that the following expression is positive:

$$
\begin{aligned}
6 & \sin (\pi / p)-8 \sin ^{3}(\pi / p)-2 \operatorname{Re}\left(-i e^{-\pi i / p-2 \pi i / k}\right) \\
& =2 \sin (3 \pi / p)+2 \sin (\pi / p+2 \pi / k) \\
& =4 \sin (\pi / 2+\pi / p-\pi / k) \sin (2 \pi / p+\pi / k) \\
& =4 \sin \left(\pi \mu_{4}\right) \sin \left(\pi \mu_{5}\right) .
\end{aligned}
$$

This is positive whenever $\mu_{4}$ and $\mu_{5}$ are both in $(0,1)$.

We know from Proposition 3.7 the complex link fractions at $z_{123}$ and $z_{134}$, and hence the order of their stabilisers. Using the explicit matrices in equations (4.7) to (4.12) we can give more information about the group structure; see Parker and Paupert [50].

Proposition 4.4. Suppose that $1 / 2-3 / p=1 / d<0$, so $p=3$, 4 or 5 . The stabiliser of $z_{123}$ is the group $\left\langle R_{1}, R_{2}\right\rangle$. This group has order $24 p^{2} /(6-p)^{2}=6 d^{2}$. It is a central extension of the orientation preserving subgroup of a $(2,3, p)$ triangle group (which has order $12 p /(6-p)=-6 d$ ) by a cyclic group of order $2 p /(6-p)=-d$.

Proof. The point $z_{123} \in \mathbf{H}_{\mathbb{C}}^{2}$ may be lifted to a vector $\mathbf{z}_{123} \in \mathbb{C}^{2,1}$. Let $U$ denote the linear subspace spanned by $\mathbf{z}_{123}$ and $U^{\perp}$ its orthogonal complement with respect to the Hermitian form $H$. By construction, $U$ is a common eigenspace of $R_{1}$ and $R_{2}$, and hence of all elements of $\left\langle R_{1}, R_{2}\right\rangle$. This means that $\left\langle R_{1}, R_{2}\right\rangle$ also preserves $U^{\perp}$ and $\mathbb{P} U^{\perp}$. Then $\mathbb{P} U^{\perp} /\left\langle R_{1}, R_{2}\right\rangle$ is the complex link of $z_{123}$. We now list the eigenvalues of some elements of $\left\langle R_{1}, R_{2}\right\rangle$. In each case, the third eigenvalue is the one that corresponds to $U$.

- $R_{1}$ has eigenvalues $e^{4 \pi i / 3 p}, e^{-2 \pi i / 3 p}, e^{-2 \pi i / 3 p}$,

- $R_{2}$ has eigenvalues $e^{4 \pi i / 3 p}, e^{-2 \pi i / 3 p}, e^{-2 \pi i / 3 p}$,

- $R_{1} R_{2}$ has eigenvalues $-e^{2 \pi i / 3+2 \pi i / 3 p},-e^{-2 \pi i / 3+2 \pi i / 3 p}, e^{-4 \pi i / 3 p}$,

- $R_{1} R_{2} R_{1}$ has eigenvalues $i e^{\pi i / p},-i e^{\pi i / p}, e^{-2 \pi i / p}$.

Observe that $R_{1}^{p}$ is the identity in $\mathrm{PU}(2,1)$ (it is $e^{-2 \pi i / 3} I$ in $\mathrm{SU}(2,1)$ ) and that $\left(R_{1} R_{2}\right)^{3}=\left(R_{1} R_{2} R_{1}\right)^{2}$ has eigenvalues $-e^{2 \pi i / p},-e^{2 \pi i / p}, e^{-4 \pi i / p}$. Hence $\left(R_{1} R_{2}\right)^{3}$ acts as the identity on $\mathbb{P} U^{\perp}$. Thus $\left\langle R_{1}, R_{2}\right\rangle$ acts on $U^{\perp}$ as the orientation preserving subgroup of a $(2,3, p)$ triangle group. Finally, $\left(R_{1} R_{2}\right)^{3}=\left(R_{1} R_{2} R_{1}\right)^{2}$ generates the centre of $\left\langle R_{1}, R_{2}\right\rangle$ and has order $2 p /(6-p)$. This proves the result. 
Proposition 4.5. Suppose that $p=6$. The stabiliser $\Gamma_{123}$ of $z_{123}$ is the group $\left\langle R_{1}, R_{2}\right\rangle$ which has infinite order. This group is a Heisenberg lattice (almost crystallographic group) obtained as a central extension of the orientation preserving subgroup of a $(2,3,6)$ triangle group $\Delta$ by an infinite cyclic group of vertical Heisenberg translations.

Proof. This is very similar to the proof of Proposition 4.4. The main difference is that $z_{123} \in \partial \mathbf{H}_{\mathbb{C}}^{2}$. We can calculate the eigenvalues as above:

- $R_{1}$ has eigenvalues $e^{2 \pi i / 9}, e^{-\pi i / 9}, e^{-\pi i / 9}$,

- $R_{2}$ has eigenvalues $e^{2 \pi i / 9}, e^{-\pi i / 9}, e^{-\pi i / 9}$,

- $R_{1} R_{2}$ has eigenvalues $e^{-2 \pi i / 9}, e^{4 \pi i / 9}, e^{-2 \pi i / 9}$,

- $R_{1} R_{2} R_{1}$ has eigenvalues $e^{2 \pi i / 3}, e^{-\pi i / 3}, e^{-\pi i / 3}$.

In this case, $\left(R_{1} R_{2}\right)^{3}=\left(R_{1} R_{2} R_{1}\right)^{2}$ is conjugate to a vertical Heisenberg translation and generates the centre of $\left\langle R_{1}, R_{2}\right\rangle$.

Proposition 4.6. Suppose that $1 / p+1 / k-1 / 2=-1 / l<0$. The stabiliser of $z_{134}$ is the group $\left\langle A_{1}, R_{2}\right\rangle$. This group has order $8 p^{2} k^{2} /(2 p+2 k-p k)^{2}=2 l^{2}$. It is a central extension of the orientation preserving subgroup of a $(2, p, k)$ triangle group (which has order $4 p k /(2 p+2 k-p k)=-2 l)$ by a cyclic group of order $2 p k /(2 p+2 k-p k)=-l$.

ProOF. This is similar to the proof of Proposition 4.4. In this case we lift $z_{134}$ to a vector $\mathbf{z}_{134}$ which spans $U$. This is a common eigenspace of $R_{2}$ and $A_{1}$. Once again we list their eigenvalues, with the eigenvalue corresponding to $U$ third.

- $R_{2}$ has eigenvalues $e^{4 \pi i / 3 p}, e^{-2 \pi i / 3 p}, e^{-2 \pi i / 3 p}$,

- $A_{1}$ has eigenvalues $e^{4 \pi i / 3 k}, e^{-2 \pi i / 3 k}, e^{-2 \pi i / 3 k}$,

- $R_{2} A_{1}$ has eigenvalues $i e^{\pi i / 3 p+\pi i / 3 k},-i e^{\pi i / 3 p+\pi i / 3 k}, e^{-2 \pi i / 3 p-2 \pi i / 3 k}$.

Hence $R_{2}^{p}$ and $A_{1}^{k}$ are the identity in $\mathrm{PU}(2,1)$. Also, $\left(R_{2} A_{1}\right)^{2}$ has eigenvalues $-e^{2 \pi i / 3 p+2 \pi i / 3 k},-e^{2 \pi i / 3 p+2 \pi i / 3 k}, e^{-4 \pi i / 3 p-4 \pi i / 3 k}$ and acts as the identity on $\mathbb{P} U^{\perp}$. Hence $\left\langle R_{2}, A_{1}\right\rangle$ acts as the orientation preserving subgroup of a $(2, p, k)$ triangle group on $\mathbb{P} U^{\perp}$. The centre of $\left\langle R_{2}, A_{1}\right\rangle$ is generated by $\left(R_{2} A_{1}\right)^{2}$ which has order $2 p k /(2 p+2 k-p k)$.

4.3. Classifying the lattices. In this section we give a classification of the lattices associated to ball quintuples with three fold symmetry.

In his seminal paper [41] Mostow considered certain complex hyperbolic triangle groups where $p$ is one of $3,4,5$. He labels these groups with a Coxeter diagram (Figure 5) and a phase shift $\varphi=\exp (\pi i t / 3)$ where $t=1 / p+2 / k-1 / 2$. Using an explicit fundamental domain, he showed that for certain values of $k$ the group $\left\langle R_{1}, R_{2}, R_{3}\right\rangle$ is a lattice in $\mathrm{SU}(2,1)$. These included the first examples of non-arithmetic complex hyperbolic lattices. We discuss these lattices in greater detail in Sections 5.1 and 5.3.

TheOREM 4.7 (Mostow [41]). Let $R_{1}, R_{2}$ and $R_{3}$ be given by (4.7), (4.8) and (4.9). Then $\left\langle R_{1}, R_{2}, R_{3}\right\rangle$ is a lattice for the following values of $p$ and $k$ :

\begin{tabular}{|l|lllllllllllllllll|}
\hline$p$ & 3 & 3 & 3 & 3 & 3 & 3 & 3 & 3 & 4 & 4 & 4 & 4 & 4 & 5 & 5 & 5 & 5 \\
$k$ & 4 & 5 & 6 & 7 & 8 & 9 & 10 & 12 & 3 & 4 & 5 & 6 & 8 & 2 & 3 & 4 & 5 \\
& $A$ & $A$ & $A$ & $N$ & $N$ & $A$ & $N$ & $A$ & $A$ & $A$ & $N$ & $N$ & $A$ & $A$ & $A$ & $N$ & $A$ \\
\hline
\end{tabular}




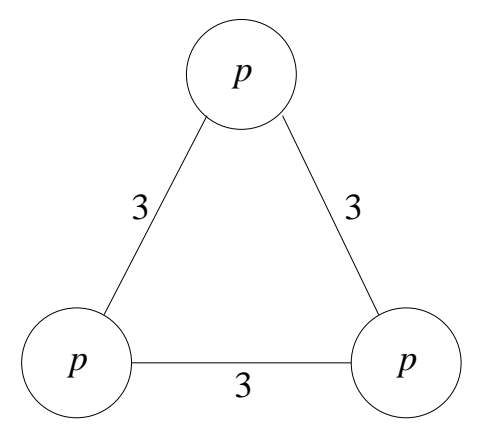

Figure 5. Mostow's Coxeter diagram for his lattices. He also specifies a phase shift $\varphi=\exp (\pi i t / 3)$ where $t=1 / p+2 / k-1 / 2$.

In the last line we have indicated which ones are arithmetic $A$ or non-arithmetic $N$.

In his thesis in 1981, Livné [35] used algebraic geometry to give a construction of 8 lattices in $\mathrm{SU}(2,1)$ parametrised by an integer $p$. We discuss these lattices in more detail in Section 5.2. In language analogous to Mostow's (which Livné did not use) Livné's groups correspond to the following values of $p$ and $k$. We remark that the groups in Theorems 4.7 and 4.8 with $p=5$ and $k=2$ are the same.

TheOREM 4.8 (Livné [35]). Let $R_{1}, R_{2}$ and $R_{3}$ be given by (4.7), (4.8) and (4.9). Then $\left\langle R_{1}, R_{2}, R_{3}\right\rangle$ is a lattice for the following values of $p$ and $k$ :

\begin{tabular}{|l|llllllll|}
\hline$p$ & 5 & 6 & 7 & 8 & 9 & 10 & 12 & 18 \\
$k$ & 2 & 2 & 2 & 2 & 2 & 2 & 2 & 2 \\
& $A$ & $A$ & $A$ & $A$ & $N$ & $A$ & $A$ & $A$ \\
\hline
\end{tabular}

In the last line we have indicated which ones are arithmetic $A$ or non-arithmetic $N$.

The groups described in Theorems 4.7 and 4.8 are all special cases of Deligne and Mostow groups with 3-fold symmetry as described in Section 3.1. They may also be described using Thurston's method using shapes of polyhedra with five vertices for which three of the cone angles are the same, as described in Section 3.2.

We now re-express Sauter's embedding theorems in terms of $p$ and $k$. We gave these results in terms of the $\mu_{i}$ in Theorem 3.11 and Corollary 3.9. Sauter's first embedding theorem, Theorem 6.1 of [57] (see also Theorem 11.22 of [12]) states:

Proposition 4.9 (Theorem 6.1 of [57]). Suppose that

$$
m \in\{4,5,6,7,8,9,10,12,15,18,24,42, \infty,-30,-12\} .
$$

Let $\Gamma$ be a Deligne-Mostow group of type $p=3, k=m$ generated by $R_{1}, A_{1}$ and $J$. Write $R_{2}=J R_{1} J^{-1}$. Then the subgroup $\Gamma^{*}$ of $\Gamma$ generated by $R_{1}^{*}=A_{1}$, $A_{1}^{*}=J\left(R_{1} R_{2}\right)^{2} J^{-1}, J^{*}=J^{-1}$ is a Deligne-Mostow group of type $p=m, k=3$. Moreover, $\Gamma^{*}$ is isomorphic to $\Gamma$ when 3 does not divide $6 m /(m-6)$.

Sauter's second embedding theorem concerns groups with $k=p / 2$, Theorem 6.2 of [57] (see also Corollary 10.18 of [12]). Recall that in this case the holonomy around $L_{j 4}$ is a complex reflection $R_{j}^{\prime}$ of angle $2 \pi / p$ satisfying the equations (4.4). 
Proposition 4.10 (Theorem 6.2 of [57]). Suppose that

$$
m \in\{5,6,7,8,9,10,12,18\} .
$$

Let $\Gamma$ be a Deligne-Mostow group of type $p=m, k=2$ generated by $R_{1}, A_{1}$ and J. Write $R_{2}=J R_{1} J^{-1}$ and $R_{3}=J^{-1} R_{1} J$. Then the group $\Gamma^{*}$ generated by $R_{1}^{*}=R_{2}^{-1} R_{1} R_{2}, R_{1}^{*}=R_{3}, J^{*}=J^{-1}$ is a Deligne-Mostow group of type $p=m$, $k=m / 2$. Moreover $\Gamma^{*}$ is isomorphic to $\Gamma$.

The following table gives a summary of the list of 46 Deligne-Mostow lattices with three fold symmetry. Of these groups, 41 satisfy the orbifold condition and the remaining 5 are related to a group satisfying the orbifold condition by a commensurability theorem (the latter are the groups in the following table for which $d$ is not an integer). All other ball quintuples with three fold symmetry lead to a non-discrete group. The specific values of all the parameters are taken from Sauter [57], but we have reordered his list. The first two columns give $p$ and $k$, as described above. Notice that this includes the case of $p=\infty$. In this case, we take $R_{1}$ to be parabolic. The subsequent columns may be deduced from the first two. We now summarise their significance.

- $l$ is the order of $A_{j}^{\prime}$. It is defined by $1 / l=1 / 2-1 / p-1 / k$. When $l$ is negative then $A_{j}^{\prime}$ is a complex reflection in a point; when $l=\infty$ then $A_{j}^{\prime}$ is parabolic; when $l$ is positive then $A_{j}^{\prime}$ is a complex reflection in a complex line with angle $2 \pi / l$. Sending the pair $\left(R_{1}, J\right)$ to $\left(R_{1}, J^{-1}\right)$ has the effect of swapping $k$ and $l$. Therefore, without loss of generality, we suppose that $1 / k \geq 1 / l$.

- $d$ is the order of $P^{3}=\left(R_{1} R_{2}\right)^{3}=\left(J A_{1}^{-1}\right)^{3}$. It is defined by $1 / d=1 / 2-3 / p$. When $d$ is negative then $P^{3}$ is a complex reflection in a point; when $d=\infty$ then $P^{3}$ is parabolic; when $d$ is positive then $P^{3}$ is a complex reflection in a complex line with angle $2 \pi / d$.

- If one of the commensurability theorems, Corollary 3.9, Corollary 3.10 or Theorem 3.11 respectively, gives a relation of the group on this line with another group then we give the pair $(p, k)$ for this new group in the appropriate one of the next three columns. For some groups more than one of these results applies and we list all of them.

- $t$ is the parameter used by Mostow. It is defined by $t=1 / p+2 / k-1 / 2$. Mostow's phase parameter $\varphi$ is defined by $\varphi^{3}=e^{\pi i t}$.

- $\mu_{i}$ for $i=1,2, \ldots, 5$ are the elements of a ball quintuple. They are

$$
\mu_{1}=\mu_{2}=\mu_{3}=\frac{1}{2}-\frac{1}{p}, \quad \mu_{4}=\frac{1}{2}+\frac{1}{p}-\frac{1}{k}, \quad \mu_{5}=\frac{2}{p}+\frac{1}{k} .
$$

Because of the three fold symmetry, $\mu_{1}=\mu_{2}=\mu_{3}$ and so we only write $\mu_{1}$ in the table.

- The last column indicates whether the lattice is arithmetic $A$ or nonarithmetic $N$. Combining the information in this table and the table at the end of section 3.3, we see that there are (at most) 15 commensurability classes of arithmetic Deligne-Mostow lattices and (at most) 9 commensurability classes of non-arithmetic Deligne-Mostow lattices. Representatives of the arithmetic classes are the lattices labelled $(a)$ and (b) from the previous table and the lattices corresponding to the following 
values of $(p, k)$ :

$$
\begin{aligned}
& (3,4),(3,5),(3,6),(3,9),(3,12),(4,4),(4,8), \\
& (5,2),(5,5),(6,6),(7,2),(10,2),(12,2) .
\end{aligned}
$$

Representatives of the non-arithmetic classes are the lattice labelled $(c)$ and the lattices corresponding to the following values of $(p, k)$ :

$$
(3,7),(3,8),(3,10),(4,5),(4,6),(5,4),(6,4),(9,2) .
$$

The rows of the table are grouped as follows. There are six blocks divided by horizontal lines. We give a fundamental domain and a presentation for each of the groups in the first three blocks, except for $p=5$ and $k=5 / 2$. The groups with an entry in one of the commensurability columns are isomorphic to subgroups of a group in the first three blocks. Hence, in principle, we could produce a fundamental domain and presentation for such groups. We now describe the different blocks.

- In the first block $1 / l \leq 0$ and $1 / d \leq 0$. This means that $A_{j}^{\prime}$ and $P^{3}$ are complex reflections in a point or are parabolic. Hence these groups are Mostow groups of the first type. We study these groups (except the case $p=5, k=5 / 2)$ in Section 5.1.

- In the second block $1 / l>0$ and $1 / d \leq 0$. This means that $A_{j}^{\prime}$ is a complex reflection in a complex line and $P^{3}$ is a complex reflection in a point or is parabolic. These groups are Mostow groups of the second type and we study them in detail in Section 5.3.

- In the third block $1 / l<0$ and $1 / d>0$. Thus $A_{j}^{\prime}$ is a complex reflection in a point and $P^{3}$ is a complex reflection in a line. These groups are of Livné type and are studied in Section 5.2

- In the fourth block $1 / l$ and $1 / d$ are both positive. Indeed, $k=3$ and $l=3 d$. In this case, $A_{j}^{\prime}$ and $P^{3}$ are both complex reflections in complex lines. Each of these groups is related to one of the previous groups by Theorem 3.11, or equivalently Proposition 4.9.

- In the fifth block $1 / l$ and $1 / d$ are again both positive. Indeed, one of $k, l$ is $p / 2$ and the other is $d$. These groups correspond to ball quintuples where four of the $\mu_{j}$ are the same. In this case, $A_{j}^{\prime}$ and $P^{3}$ are both complex reflections in a complex lines. Each of these groups is related to a group of Livné type by Corollary 3.9 or equivalently Proposition 4.10. This also applies to the groups with $p=5, k=5 / 2 ; p=6, k=3$ and $p=18, k=3$.

- The final block contains one group. This is an index two subgroup of the Gauss-Picard modular group. This group is related to the group with $p=k=4$ using Corollary 3.10. This group is considered in Section 5.4

The first two blocks are related to Tables 1 and 2 on page 248 of Mostow [41]. The first difference is that Mostow only considers $p=3,4,5$. We allow $p=6$ as well. In Table 1 Mostow considers the condition $0 \leq t \leq 1 / 2-1 / p$. The first of these inequalities translates to $1 / l \leq 1 / k$ and the second to $1 / l \geq 0$. Thus Table 1 corresponds to our second block but also includes those groups in the first block with $l=\infty$ (that is $1 / l=0$ ). In Table 2 Mostow considers the condition $1 / 2-1 / p<t<3(1 / 2-1 / p)$. The first of these inequalities corresponds to $1 / l<0$ and the second to $\mu_{5}<1$. This means that Table 2 corresponds to our first block. Moreover, in the remark following Table 2, Mostow indicates that the group with $p=5$ and $k=5 / 2$ can be included in this block. 


\begin{tabular}{|c|c|c|c|c|c|c|c|c|c|c|c|}
\hline$p$ & $k$ & l & $d$ & 3.9 & 3.10 & 3.11 & $t$ & $\mu_{1}$ & $\mu_{4}$ & $\mu_{5}$ & \\
\hline 3 & 4 & -12 & -2 & & & & $\frac{1}{3}$ & $\frac{1}{6}$ & $\frac{7}{12}$ & $\frac{11}{12}$ & $A$ \\
\hline 3 & 5 & -30 & -2 & & & & $\frac{7}{30}$ & $\frac{1}{6}$ & $\frac{19}{30}$ & $\frac{13}{15}$ & $A$ \\
\hline 3 & 6 & $\infty$ & -2 & & & & $\frac{10}{6}$ & $\frac{1}{6}$ & $\frac{2}{3}$ & $\frac{5}{6}$ & $A$ \\
\hline 4 & 3 & -12 & -4 & & & $(3,4)$ & $\frac{5}{12}$ & & $\frac{5}{12}$ & $\frac{5}{6}$ & $A$ \\
\hline 4 & 4 & $\infty$ & -4 & & & & $\frac{1}{4}$ & $\frac{1}{4}$ & $\frac{1}{2}$ & $\frac{3}{4}$ & $A$ \\
\hline 5 & 2 & -5 & -10 & & & & $\frac{7}{10}$ & $\frac{3^{4}}{10}$ & $\frac{1}{5}$ & $\frac{9^{t}}{10}$ & $A$ \\
\hline 5 & $\frac{5}{2}$ & -10 & -10 & $(5,2)$ & & & $\frac{10}{2}$ & $\frac{3}{10}$ & $\frac{3}{10}$ & $\frac{4}{5}$ & $A$ \\
\hline 5 & 3 & -30 & -10 & & & $(3,5)$ & $\frac{11}{30}$ & $\frac{3}{10}$ & $\frac{11}{30}$ & $\frac{11}{15}$ & $A$ \\
\hline 6 & 2 & -6 & $\infty$ & & & & $\frac{2}{3}$ & $\frac{1}{3}$ & $\frac{1}{6}$ & $\frac{5}{6}$ & $A$ \\
\hline 6 & 3 & $\infty$ & $\infty$ & $(6,2)$ & & $(3,6)$ & $\frac{1}{3}$ & & $\frac{1}{3}$ & $\frac{2}{3}$ & $A$ \\
\hline 3 & 7 & 42 & -2 & & & & $\frac{5}{42}$ & $\frac{1}{6}$ & $\frac{29}{42}$ & $\frac{17}{21}$ & $N$ \\
\hline 3 & 8 & 24 & -2 & & & & $\frac{1}{12}$ & $\frac{1}{6}$ & $\frac{17}{24}$ & $\frac{19}{24}$ & $N$ \\
\hline 3 & 9 & 18 & -2 & & & & $\frac{1}{18}$ & $\frac{1}{6}$ & $\frac{13}{18}$ & $\frac{7}{9}$ & $A$ \\
\hline 3 & 10 & 15 & -2 & & & & $\frac{1}{30}$ & $\frac{1}{6}$ & $\frac{19}{15}$ & $\frac{23}{30}$ & $N$ \\
\hline 3 & 12 & 12 & -2 & & & & 0 & $\frac{1}{6}$ & $\frac{3}{4}$ & $\frac{3}{4}$ & $A$ \\
\hline 4 & 5 & 20 & -4 & & & & $\frac{3}{20}$ & $\frac{1}{4}$ & $\frac{11}{20}$ & $\frac{7}{10}$ & $N$ \\
\hline 4 & 6 & 12 & -4 & & & & $\frac{1}{12}$ & & $\frac{7}{12}$ & $\frac{2}{3}$ & $N$ \\
\hline 4 & 8 & 8 & -4 & & & & 0 & & $\frac{5}{8}$ & $\frac{5}{8}$ & $A$ \\
\hline 5 & 4 & 20 & -10 & & & & $\frac{1}{5}$ & $\frac{3}{10}$ & $\frac{9}{20}$ & $\frac{13}{20}$ & $N$ \\
\hline 5 & 5 & 10 & -10 & & & & $\frac{1}{10}$ & $\frac{3}{10}$ & $\frac{1}{2}$ & $\frac{3}{5}$ & $A$ \\
\hline 6 & 4 & 12 & $\infty$ & & & & $\frac{10}{6}$ & $\frac{1}{3}$ & $\frac{5}{12}$ & $\frac{7}{12}$ & $N$ \\
\hline 6 & 6 & 6 & $\infty$ & & & & 0 & $\frac{1}{3}$ & $\frac{1}{2}$ & $\frac{1}{2}$ & $A$ \\
\hline 7 & 2 & -7 & 14 & & & & $\frac{9}{14}$ & & & $\frac{11}{14}$ & $A$ \\
\hline 8 & 2 & -8 & 8 & & & & $\frac{5}{8}$ & $\frac{3}{8}$ & $\frac{1}{8}$ & $\frac{3}{4}$ & $A$ \\
\hline 9 & 2 & -9 & 6 & & & & $\frac{11}{18}$ & $\frac{7}{18}$ & $\frac{i}{9}$ & $\frac{13}{18}$ & $N$ \\
\hline 10 & 2 & -10 & 5 & & & & $\frac{30}{5}$ & $\frac{8}{5}$ & $\frac{1}{10}$ & $\frac{7}{10}$ & $A$ \\
\hline 12 & 2 & -12 & 4 & & & & $\frac{7}{12}$ & $\frac{5}{12}$ & $\frac{1}{12}$ & $\frac{2}{3}$ & $A$ \\
\hline 18 & 2 & -18 & 3 & & & & $\frac{5}{9}$ & $\frac{4}{9}$ & $\frac{1}{18}$ & $\frac{11}{18}$ & $A$ \\
\hline 7 & 3 & 42 & 14 & & & $(3,7)$ & $\frac{13}{42}$ & $\frac{5}{14}$ & $\frac{13}{42}$ & $\frac{13}{21}$ & $N$ \\
\hline 8 & 3 & 24 & 8 & & & $(3,8)$ & $\frac{7}{24}$ & $\frac{3}{8}$ & $\frac{7}{24}$ & $\frac{4}{12}$ & $N$ \\
\hline 9 & 3 & 18 & 6 & & & $(3,9)$ & $\frac{5}{18}$ & $\frac{7}{18}$ & $\frac{5}{18}$ & $\frac{5}{9}$ & $A$ \\
\hline 10 & 3 & 15 & 5 & & & $(3,10)$ & $\frac{4}{15}$ & $\frac{2}{5}$ & $\frac{4}{15}$ & $\frac{8}{15}$ & $N$ \\
\hline 12 & 3 & 12 & 4 & & & $(3,12)$ & $\frac{10}{4}$ & $\frac{5}{12}$ & $\frac{1}{4}$ & $\frac{1}{2}$ & $A$ \\
\hline 15 & 3 & 10 & $\frac{10}{3}$ & & & $(3,15)$ & $\frac{7}{30}$ & $\frac{13}{30}$ & $\frac{7}{30}$ & $\frac{7}{15}$ & $N$ \\
\hline 18 & 3 & 9 & 3 & $(18,2)$ & & $(3,18)$ & $\frac{2}{9}$ & $\frac{4}{9}$ & $\frac{2}{9}$ & $\frac{4}{9}$ & $A$ \\
\hline 24 & 3 & 8 & $\frac{8}{3}$ & & & $(3,24)$ & $\frac{5}{24}$ & $\frac{11}{24}$ & $\frac{5}{24}$ & $\frac{5}{12}$ & $N$ \\
\hline 42 & 3 & 7 & $\frac{7}{3}$ & & & $(3,42)$ & $\frac{4}{21}$ & $\frac{10}{21}$ & $\frac{4^{4}}{21}$ & $\frac{8}{21}$ & $N$ \\
\hline$\infty$ & 3 & 6 & 2 & & & $(3, \infty)$ & $\frac{1}{6}$ & $\frac{1}{2}$ & $\frac{1}{6}$ & $\frac{1}{3}$ & $A$ \\
\hline-30 & 3 & 5 & $\frac{5}{3}$ & & & $(3,-30)$ & $\frac{2}{15}$ & $\frac{8}{15}$ & $\frac{2}{15}$ & $\frac{4}{15}$ & $A$ \\
\hline-12 & 3 & 4 & $\frac{4}{3}$ & & & $(3,-12)$ & $\frac{1}{12}$ & $\frac{7}{12}$ & $\frac{1}{12}$ & $\frac{1}{6}$ & $A$ \\
\hline 7 & $\frac{7}{2}$ & 14 & 14 & $(7,2)$ & & & $\frac{3}{14}$ & & $\frac{5}{14}$ & $\frac{4}{7}$ & $A$ \\
\hline 8 & 4 & 8 & 8 & $(8,2)$ & $(4,8)$ & & $\frac{1}{8}$ & $\frac{3}{8}$ & $\frac{3}{8}$ & $\frac{1}{2}$ & $A$ \\
\hline 9 & $\frac{9}{2}$ & 6 & 6 & $(9,2)$ & & & $\frac{1}{18}$ & & $\frac{7}{18}$ & $\frac{4}{9}$ & $N$ \\
\hline 10 & 5 & 5 & 5 & $(10,2)$ & & & 0 & $\frac{2}{5}$ & $\frac{2}{5}$ & $\frac{2}{5}$ & $A$ \\
\hline 12 & 4 & 6 & 4 & $(12,2)$ & & & $\frac{1}{12}$ & $\frac{5}{12}$ & & $\frac{5}{12}$ & $A$ \\
\hline$\infty$ & 4 & 4 & 2 & & $(4,4)$ & & 0 & $\frac{1}{2}$ & $\frac{1}{4}$ & $\frac{1}{4}$ & $A$ \\
\hline
\end{tabular}




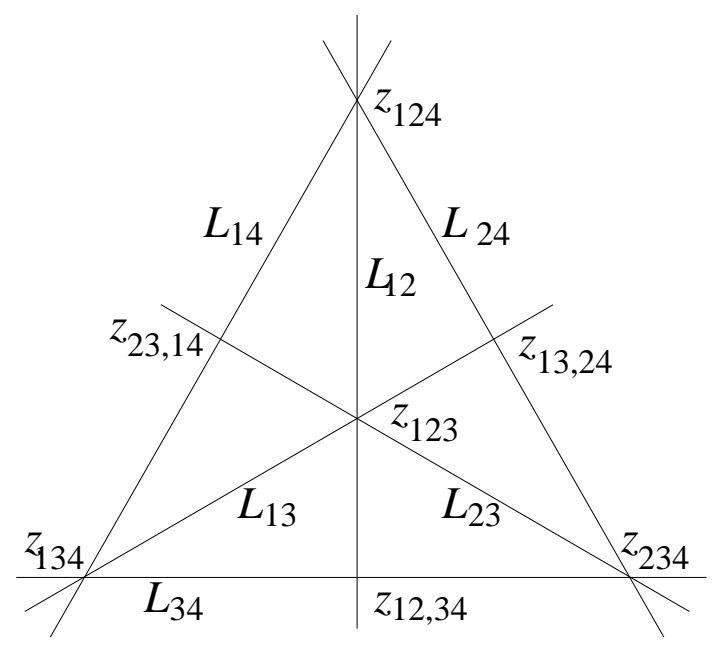

FiguRE 6 . The line arrangement when $l \leq 0$ and $d \leq 0$ corresponding to Mostow groups of the first type.

\section{Fundamental domains}

In this section we summarise the construction of fundamental domains for Mostow groups and Livné groups. We indicate how to use Poincaré's theorem to give a presentation for the lattice and we show how to calculate the orbifold Euler characteristic.

5.1. Mostow groups of the first type. In this section we consider the groups where $l \leq 0$ and $d \leq 0$. The values of $p$ and $k$, together with $l$ and $d$ are:

\begin{tabular}{|l|rrrrrrrrr|}
\hline$p$ & 3 & 3 & 3 & 4 & 4 & 5 & 5 & 6 & 6 \\
$k$ & 4 & 5 & 6 & 3 & 4 & 2 & 3 & 2 & 3 \\
$-d$ & 2 & 2 & 2 & 4 & 4 & 10 & 10 & $\infty$ & $\infty$ \\
$-l$ & 12 & 30 & $\infty$ & 12 & $\infty$ & 5 & 30 & 6 & $\infty$ \\
\hline
\end{tabular}

In this case the four lines involving $v_{5}$ in the complex line arrangement described above have collapsed to a point. Instead there are four zero dimensional strata $z_{123}$, $z_{124}, z_{134}$ and $z_{234}$, where the stratum $z_{i j k}=L_{i j} \cap L_{j k} \cap L_{k i}$. We illustrate this in Figure 6. Algebraic geometers call this line arrangement the complete quadrilateral. In [23] Hirzebruch gives a non-technical description of the algebraic surface corresponding to one of these lattices; see also [24] or [6]. An explicit relation between Hirzebruch's construction and Mostow's construction is given by Yamazaki and Yoshida [67].

For these groups Mostow constructed a fundamental domain in [41]. In his thesis [8], Richard Kena Boadi gives a similar construction along the lines of that in [47]. In Boadi's construction, the fundamental domain $D$ is made up of two four dimensional simplices glued along a common three dimensional face. The vertices of the simplices are the strata $z_{123}, z_{124}, z_{134}, z_{234}, z_{23,14}$ and $z_{13,24}$. The boundary of $D$ is made up of eight three dimensional simplices. Each side is contained in a bisector and they are identified by the side pairing maps $J, P, R_{1}$ and $R_{2}$. We use these maps to label the sides. Therefore $J: S(J) \longrightarrow S\left(J^{-1}\right)$ and so on. These maps are illustrated in Figure 7. All the one dimensional simplices in the boundary 

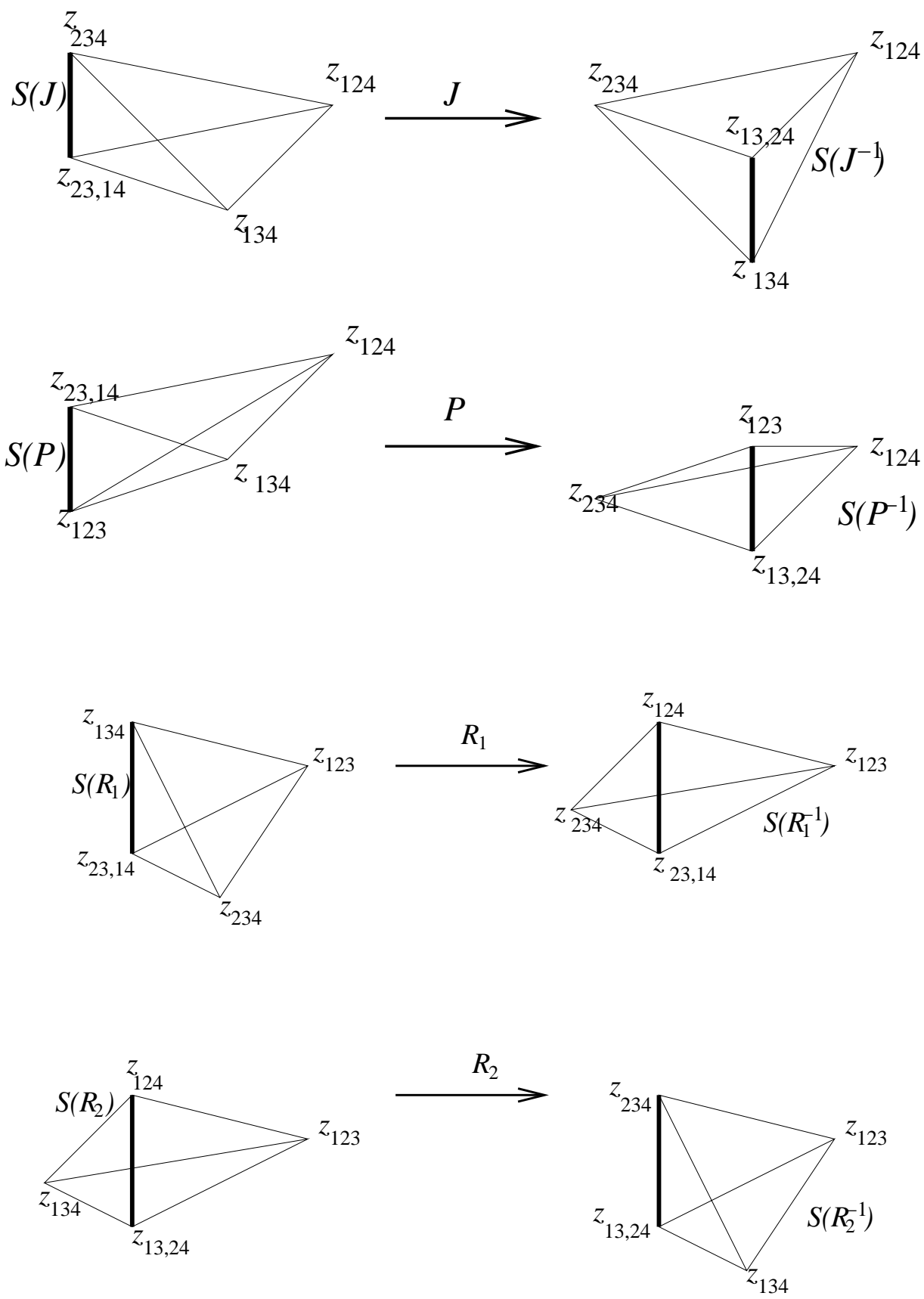

Figure 7. The sides of the Mostow polyhedron and side pairings. The bold lines denote the spines of the bisectors. 
of each side are arcs of geodesics, one of which is contained in the spine of the bisector. Both the 2-simplices containing this 1-simplex are contained in meridians of the bisector. One of the other 2-simplices in the boundary of the face is a triangle in one of the complex lines $L_{14}, L_{24}, L_{23}$ or $L_{13}$. These complex lines are the fixed lines of $A_{1}, A_{2}, R_{1}$ and $R_{2}$ respectively.

We can use the complex hyperbolic version of Poincaré's polyhedron theorem to give a presentation for the group in this case. The cycle relations are

\begin{tabular}{|l|l|l|}
\hline Face & Cycle element & Cycle relation \\
\hline$F\left(J, J^{-1}\right)=S(J) \cap S\left(J^{-1}\right)$ & $J$ & $J^{3}=I$ \\
$F\left(R_{1}, R_{2}^{-1}\right)=S\left(R_{1}\right) \cap S\left(R_{2}^{-1}\right)$ & $R_{2} P^{-1} R_{1}$ & $R_{2} P^{-1} R_{1}=1$ \\
$F\left(P, R_{1}\right)=S(P) \cap S\left(R_{1}\right)$ & $P^{-1} R_{2}^{-1} P R_{1}$ & $P^{-1} R_{2}^{-1} P R_{1}=1$ \\
$F\left(J, R_{1}\right)=S(J) \cap S\left(R_{1}\right)$ & $J^{-1} R_{2}^{-1} J R_{1}$ & $J^{-1} R_{2}^{-1} J R_{1}=1$ \\
$F(J, P)=S(J) \cap S(P)$ & $P^{-1} J$ & $\left(P^{-1} J\right)^{k}=1$ \\
$F\left(R_{1}, R_{1}^{-1}\right)=S\left(R_{1}\right) \cap S\left(R_{1}^{-1}\right)$ & $R_{1}$ & $R_{1}^{p}=1$ \\
$F\left(R_{2}, R_{2}^{-1}\right)=S\left(R_{2}\right) \cap S\left(R_{2}^{-1}\right)$ & $R_{2}$ & $R_{2}^{p}=1$ \\
\hline
\end{tabular}

This means that the group has the following presentation:

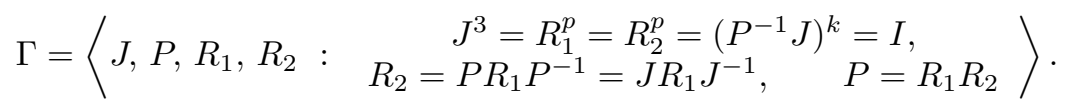

Using $A_{1}=P^{-1} J$, we may rewrite this presentation as

$$
\left\langle J, R_{1}, A_{1},: \begin{array}{l}
J^{3}=R_{1}^{p}=A_{1}^{k}=1 \\
A_{1}=\left(J R_{1}^{-1} J\right)^{2}, A_{1} R_{1}=R_{1} A_{1}
\end{array}\right\rangle
$$

The case of $p=6$ and $k=2$ was considered by Falbel and Parker in [17]. This group is the Eisenstein-Picard modular group $\mathrm{PU}\left(2,1 ; \mathcal{O}_{3}\right)$. In $[\mathbf{1 7}]$ the standard off-diagonal Hermitian form is used. In this case $z_{123}$ is the point at infinity in the Siegel domain model of complex hyperbolic space. Writing $\omega=(-1+i \sqrt{3}) / 2$, the corresponding matrices are

$$
J=\left[\begin{array}{ccc}
0 & 0 & 1 \\
0 & -\omega & \omega \\
1 & 1 & \omega
\end{array}\right], \quad R_{1}=\left[\begin{array}{ccc}
1 & 0 & 0 \\
0 & -\bar{\omega} & 0 \\
0 & 0 & 1
\end{array}\right], \quad A_{1}=\left[\begin{array}{ccc}
0 & 0 & \omega \\
0 & -\omega & 0 \\
\omega & 0 & 0
\end{array}\right]
$$

Falbel and Parker construct the Ford domain and use Poincaré's theorem to give a presentation of this group, which is essentially the one given above. Other presentations are given in Alezais [1] and Holzapfel [28].

By examining the fundamental domain $D$ and its side pairings, we can calculate the orbifold Euler characteristic $\chi\left(\mathbf{H}_{\mathbb{C}}^{2} / \Gamma\right)$. This is calculated in the same way as the standard Euler characteristic of a cell complex. Namely one takes the alternating sum of the number of cells of each dimension. The main difference is that now a cell is counted with a weight, which is the reciprocal of the order of its stabiliser. In the first block of the following table we list the orbits of vertices. The stabiliser given in the second column is the stabiliser of the first point in the list in the first column. Similarly in the second block we list the orbits on one dimensional simplices with the stabiliser of the first one. These one dimensional simplices are given as a pair of vertices in the obvious way. The next blocks do the same thing for the 2 -simplices, 
3 -simplices and finally the only four cell, the whole of $D$.

\begin{tabular}{|l|l|l|}
\hline Orbit & Stabiliser & Order \\
\hline$z_{23,14}, z_{13,24}$ & $\left\langle R_{1}, A_{1}\right\rangle$ & $k p$ \\
$z_{134}, z_{124}, z_{234}$ & $\left\langle R_{2}, A_{1}\right\rangle$ & $2 l^{2}$ \\
$z_{123}$ & $\left\langle R_{1}, R_{2}\right\rangle$ & $6 d^{2}$ \\
\hline$\left(z_{23,14}, z_{123}\right),\left(z_{13,24}, z_{123}\right)$ & $\left\langle R_{1}\right\rangle$ & $p$ \\
$\left(z_{234}, z_{123}\right),\left(z_{134}, z_{123}\right),\left(z_{124}, z_{123}\right)$ & $\left\langle R_{1}\right\rangle$ & $p$ \\
$\left\{\begin{array}{l}\left(z_{23,14}, z_{124}\right),\left(z_{23,14}, z_{134}\right), \\
\left(z_{12,24}, z_{124}\right),\left(z_{13,24}, z_{234}\right)\end{array}\right\}$ & $\left\langle A_{1}\right\rangle$ & $k$ \\
$\left(z_{23,14}, z_{234}\right),\left(z_{13,24}, z_{134}\right)$ & $\left\langle R_{1}\right\rangle$ & $p$ \\
$\left(z_{124}, z_{234}\right),\left(z_{234}, z_{134}\right),\left(z_{134}, z_{124}\right)$ & $\left\langle R_{1} J\right\rangle$ & $2 k$ \\
\hline$F(J, P), F\left(J^{-1}, P^{-1}\right)$ & $\left\langle A_{1}\right\rangle$ & $k$ \\
$\left\{\begin{array}{l}F\left(J, R_{1}\right), F\left(J, R_{1}^{-1}\right), \\
F\left(J^{-1}, R_{2}\right), F\left(J^{-1}, R_{2}^{-1}\right)\end{array}\right\}$ & 1 & 1 \\
$\left\{\begin{array}{l}\left.F, R_{1}\right), F\left(P, R_{1}^{-1}\right), \\
F\left(P^{-1}, R_{2}\right), F\left(P^{-1}, R_{2}^{-1}\right)\end{array}\right\}$ & 1 & \\
$F\left(P, R_{2}\right), F\left(P^{-1} R_{1}^{-1}\right), F\left(R_{1}, R_{2}^{-1}\right)$ & 1 & 1 \\
$F\left(J, J^{-1}\right)$ & $\langle J\rangle$ & 1 \\
$F\left(R_{1}, R_{1}^{-1}\right)$ & $\left\langle R_{1}\right\rangle$ & $p$ \\
$F\left(R_{2}, R_{2}^{-1}\right)$ & $\left\langle R_{2}\right\rangle$ & $p$ \\
\hline$S(J), S\left(J^{-1}\right)$ & 1 & 1 \\
$S(P), S\left(P^{-1}\right)$ & 1 & 1 \\
$S\left(R_{1}\right), S\left(R_{1}^{-1}\right)$ & 1 & 1 \\
$S\left(R_{2}\right), S\left(R_{2}^{-1}\right)$ & 1 & 1 \\
\hline$D$ & & 1 \\
\hline
\end{tabular}

Then the orbifold Euler characteristic $\chi\left(\mathbf{H}_{\mathbb{C}}^{2} / \Gamma\right)$ may be calculated by summing the entries in the last column of the previous table, with alternating signs depending on the dimension, that is depending on which block we are in. Using $d=2 p /(p-6)$ we obtain:

$$
\begin{aligned}
\chi\left(\mathbf{H}_{\mathbb{C}}^{2} / \Gamma\right)= & \frac{1}{k p}+\frac{1}{2 l^{2}}+\frac{1}{6 d^{2}}-\frac{1}{p}-\frac{1}{p}-\frac{1}{k}-\frac{1}{p}-\frac{1}{2 k} \\
& +\frac{1}{k}+1+1+1+\frac{1}{3}+\frac{1}{p}+\frac{1}{p}-1-1-1-1+1 \\
= & \frac{1}{k p}+\frac{1}{8}-\frac{1}{2 k}-\frac{1}{2 p}+\frac{1}{2 k^{2}}+\frac{1}{k p}+\frac{1}{2 p^{2}} \\
& \quad+\frac{1}{24}-\frac{1}{2 p}+\frac{3}{2 p^{2}}-\frac{1}{p}-\frac{1}{2 k}+\frac{1}{3} \\
= & \frac{1}{2}\left(2\left(\frac{1}{2}-\frac{1}{p}\right)-\frac{1}{k}\right)^{2} .
\end{aligned}
$$

Using $2 / k=1 / 2-1 / p+t$ we see that this agrees with Sauter Theorem 5.2 of [57]; see also [69] for computations of Euler characteristics. (We have $J$ in our group so the volume of Sauter's $\Omega$ is three times ours; see the discussion on page 361 of $[57]$. 


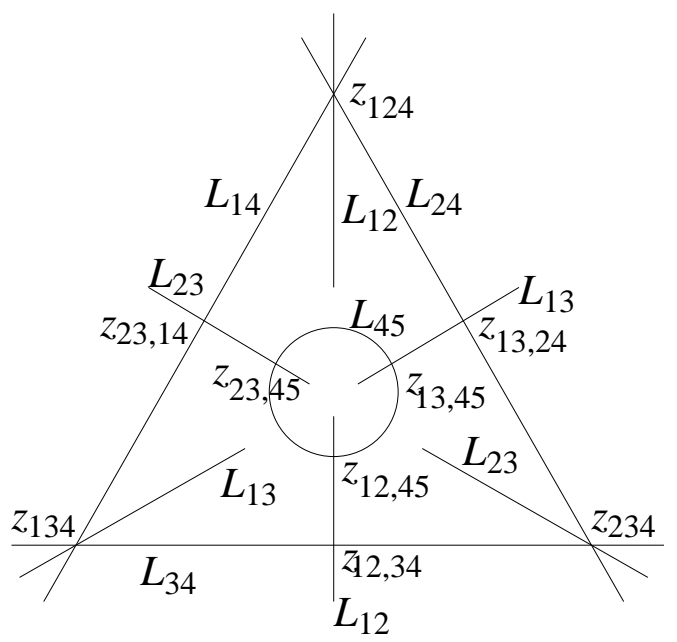

FiguRE 8. The line arrangement when $l \leq 0$ and $d>0$ corresponding to Livné groups.

5.2. Livné groups. In this section we consider the groups where $l \leq 0$ and $d>0$. The values of $p$ and $k$ together with $l$ and $d$ are:

\begin{tabular}{|l|rrrrrr|}
\hline$p$ & 7 & 8 & 9 & 10 & 12 & 18 \\
$k$ & 2 & 2 & 2 & 2 & 2 & 2 \\
$d$ & 14 & 8 & 6 & 5 & 4 & 3 \\
$-l$ & 7 & 8 & 9 & 10 & 12 & 18 \\
\hline
\end{tabular}

Since $l \leq 0$, the three lines $L_{15}, L_{25}$ and $L_{35}$ have each collapsed to a point as in the previous section. Again they are replaced by zero dimensional strata $z_{234}, z_{134}$ and $z_{124}$. However, since $d>0$ the line $L_{45}$ has not collapsed. We illustrate this in Figure 8 which should be compared with Figure 5.4 of Sauter [57].

These groups were constructed by Livné in [35]. Parker [47] constructed a fundamental domain for them. Combinatorially, this fundamental domain is very similar to that described in the previous section. Again, the polyhedron has eight sides labelled by the side pairing maps $J, P, R_{1}$ and $R_{2}$. The main difference is that the vertex $z_{123}$ has been truncated with a triangle contained in the complex line $L_{45}$. This triangle has vertices $z_{23,45}, z_{13,45}$ and $z_{12,45}$. The fundamental domain still has eight codimension 1 sides and the side pairing maps are the same. However, the sides are not all 3-simplices. Each side with a vertex $z_{123}$ in the previous section has this vertex blown up either to a 1-simplex or a 2-simplex. The stabiliser of $z_{23,45}$ is the direct product of a cyclic group of order $p$ generated by $R_{1}$ and a cyclic group of order $d=2 p /(p-6)$ generated by $\left(R_{1} R_{2}\right)^{3}$. Thus it has order $d p$. We can find the stabilisers of $z_{13,45}$ and $z_{12,45}$ similarly.

We can use Proposition 4.6 to describe the structure of the stabiliser of $z_{134}$. Because $k=2$ we see that $-1 / l=1 / p$ and the stabiliser of $z_{134}$ is a central extension of a dihedral group of order $2 p$ by a cyclic group of order $p$ generated by $\left(R_{2} A_{1}\right)^{2}$.

In Parker's construction [47] the fundamental domain has eight sides, each of which is contained in a bisector. The 1-simplices in the boundary of each side are all arcs of geodesics. Two of the two dimensional simplices in the boundary of each of $S(P)$ and $S\left(P^{-1}\right)$ are contained in complex lines. These are the fixed point sets 

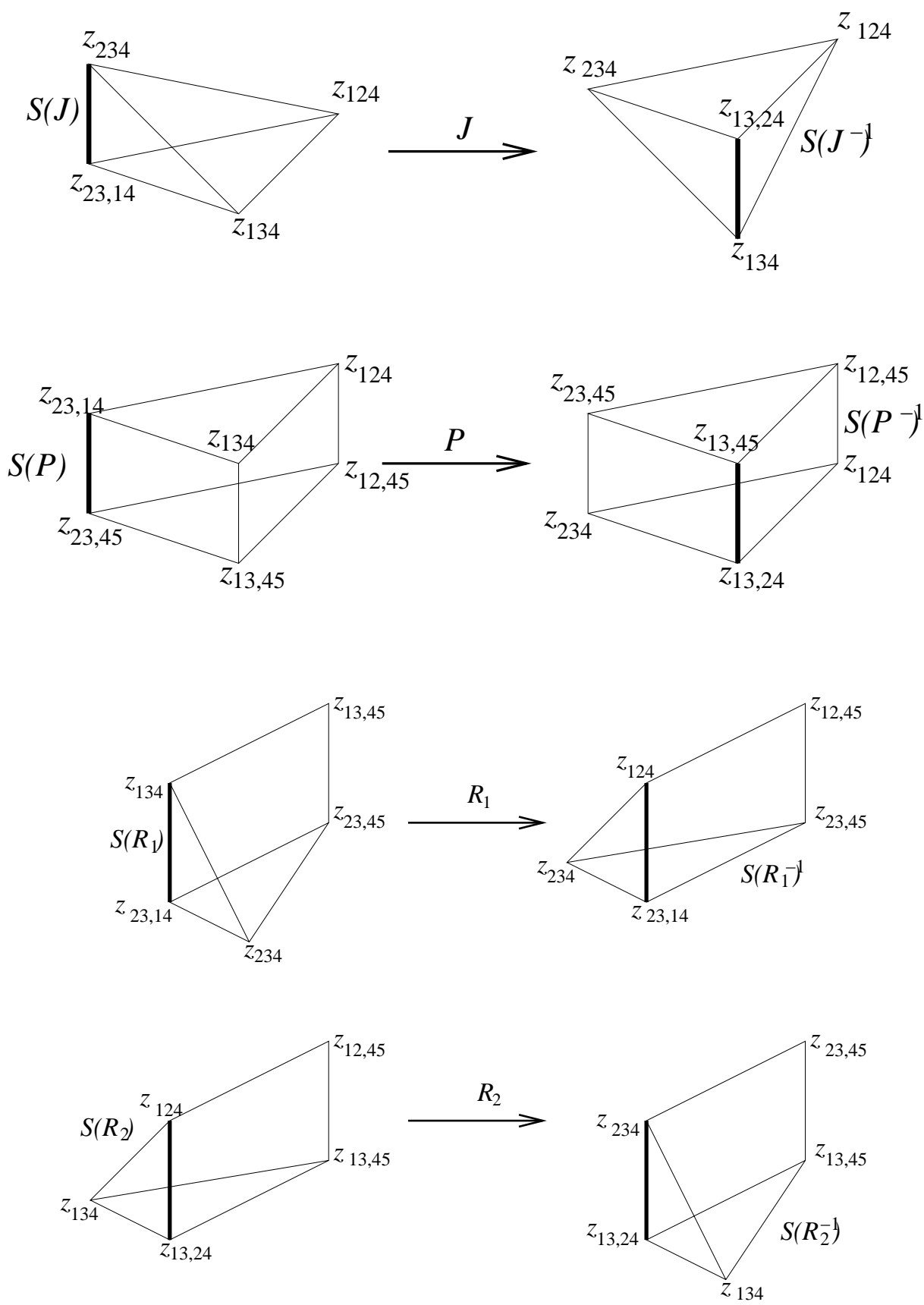

Figure 9. The sides of the Livné polyhedron and side pairings. The bold lines denote the spines of the bisectors. 
$L_{14}, L_{24}$ and $L_{45}$ of $A_{1}, A_{2}$ and $P^{3}$ respectively. All the other sides have one of the 2-simplices in their boundary contained in complex lines, namely the complex lines $L_{14}, L_{24}, L_{23}$ and $L_{13}$ fixed by $A_{1}, A_{2}, R_{1}$ and $R_{2}$.

Proceeding as above, we can use Poincaré's polyhedron theorem to give a presentation for the group. The cycle relations are

\begin{tabular}{|l|l|l|}
\hline Face & Cycle element & cycle relation \\
\hline$F\left(J, J^{-1}\right)=S(J) \cap S\left(J^{-1}\right)$ & $J$ & $J^{3}=I$ \\
$F\left(R_{1}, R_{2}^{-1}\right)=S\left(R_{1}\right) \cap S\left(R_{2}^{-1}\right)$ & $R_{2} P^{-1} R_{1}$ & $R_{2} P^{-1} R_{1}=1$ \\
$F\left(P, R_{1}\right)=S(P) \cap S\left(R_{1}\right)$ & $P^{-1} R_{2}^{-1} P R_{1}$ & $P^{-1} R_{2}^{-1} P R_{1}=1$ \\
$F\left(J, R_{1}\right)=S(J) \cap S\left(R_{1}\right)$ & $J^{-1} R_{2}^{-1} J R_{1}$ & $J^{-1} R_{2}^{-1} J R_{1}=1$ \\
$F(J, P)=S(J) \cap S(P)$ & $P^{-1} J$ & $\left(P^{-1} J\right)^{2}=1$ \\
$F\left(R_{1}, R_{1}^{-1}\right)=S\left(R_{1}\right) \cap S\left(R_{1}^{-1}\right)$ & $R_{1}$ & $R_{1}^{p}=1$ \\
$F\left(R_{2}, R_{2}^{-1}\right)=S\left(R_{2}\right) \cap S\left(R_{2}^{-1}\right)$ & $R_{2}$ & $R_{2}^{p}=1$ \\
$F\left(P, P^{-1}\right)=S(P) \cap S\left(P^{-1}\right)$ & $P$ & $P^{3 d}=1$ \\
\hline
\end{tabular}

This means that we have the following presentation:

$$
\Gamma=\left\langle J, P, R_{1}, R_{2}: \begin{array}{c}
J^{3}=P^{3 d}=R_{1}^{p}=R_{2}^{p}=\left(P^{-1} J\right)^{2}=I, \\
R_{2}=P R_{1} P^{-1}=J R_{1} J^{-1}, \quad P=R_{1} R_{2}
\end{array}\right\rangle .
$$

Using $A_{1}=P^{-1} J$ we may rewrite this presentation as:

$$
\left\langle J, R_{1}, A_{1},: \begin{array}{l}
J^{3}=R_{1}^{p}=A_{1}^{2}=\left(J A_{1}^{-1}\right)^{3 d}=1 \\
A_{1}=\left(J R_{1}^{-1} J\right)^{2}, A_{1} R_{1}=R_{1} A_{1}
\end{array}\right\rangle
$$

Once again, we can calculate the orbifold Euler characteristic.

\begin{tabular}{|l|l|l|}
\hline Orbit & Stabiliser & Order \\
\hline$z_{23,14}, z_{13,24}$ & $\left\langle R_{1}, A_{1}\right\rangle$ & $2 p$ \\
$z_{134}, z_{124}, z_{234}$ & $\left\langle R_{2}, A_{1}\right\rangle$ & $2 p^{2}$ \\
$z_{23,45}, z_{13,45}, z_{12,45}$ & $\left\langle\left(R_{1} R_{2}\right)^{3}, R_{1}\right\rangle$ & $p d$ \\
\hline$\left(z_{23,14}, z_{23,45}\right),\left(z_{13,24}, z_{13,45}\right)$ & $\left\langle R_{1}\right\rangle$ & $p$ \\
$\left(z_{234}, z_{23,45}\right),\left(z_{134}, z_{13,45}\right),\left(z_{124}, z_{12,45}\right)$ & $\left\langle R_{1}\right\rangle$ & $p$ \\
$\left\{\left(z_{23,14}, z_{124}\right),\left(z_{23,14}, z_{134}\right),\right\}$ & $\left\langle A_{1}\right\rangle$ & 2 \\
$\left\{\left(z_{13,24}, z_{124}\right),\left(z_{13,24}, z_{234}\right)\right.$ & $\left\langle R_{1}\right\rangle$ & $p$ \\
$\left(z_{23,14}, z_{234}\right),\left(z_{13,24}, z_{134}\right)$ & $\left\langle R_{1} J\right\rangle$ & 4 \\
$\left(z_{124}, z_{234}\right),\left(z_{234}, z_{134}\right),\left(z_{134}, z_{124}\right)$ & $\left\langle R_{1} R_{2} R_{1}\right\rangle$ & $2 d$ \\
$\left(z_{23,45}, z_{13,45}\right),\left(z_{13,45}, z_{12,45}\right),\left(z_{12,45}, z_{23,45}\right)$ & $\left\langle P^{-1} J\right\rangle$ & 2 \\
\hline$F(J, P), F\left(J^{-1}, P^{-1}\right)$ & 1 & 1 \\
$F\left(J, R_{1}\right), F\left(J, R_{1}^{-1}\right), F\left(J^{-1}, R_{2}\right), F\left(J^{-1}, R_{2}^{-1}\right)$ & 1 \\
$F\left(P, R_{1}\right), F\left(P, R_{1}^{-1}\right), F\left(P^{-1}, R_{2}\right), F\left(P^{-1}, R_{2}^{-1}\right)$ & 1 & 1 \\
$F\left(P, R_{2}\right), F\left(P^{-1} R_{1}^{-1}\right), F\left(R_{1}, R_{2}^{-1}\right)$ & 1 & 1 \\
$F\left(J, J^{-1}\right)$ & $\langle J\rangle$ & 3 \\
$F\left(R_{1}, R_{1}^{-1}\right)$ & $\left\langle R_{1}\right\rangle$ & $p$ \\
$F\left(R_{2}, R_{2}^{-1}\right)$ & $\left\langle R_{2}\right\rangle$ & $p$ \\
$F\left(P, P^{-1}\right)$ & $\langle P\rangle$ & $3 d$ \\
\hline$S(J), S\left(J^{-1}\right)$ & 1 & 1 \\
$S(P), S\left(P^{-1}\right)$ & 1 & 1 \\
$S\left(R_{1}\right), S\left(R_{1}^{-1}\right)$ & 1 & 1 \\
$S\left(R_{2}\right), S\left(R_{2}^{-1}\right)$ & 1 & 1 \\
\hline$D$ & 1 & 1 \\
\hline
\end{tabular}




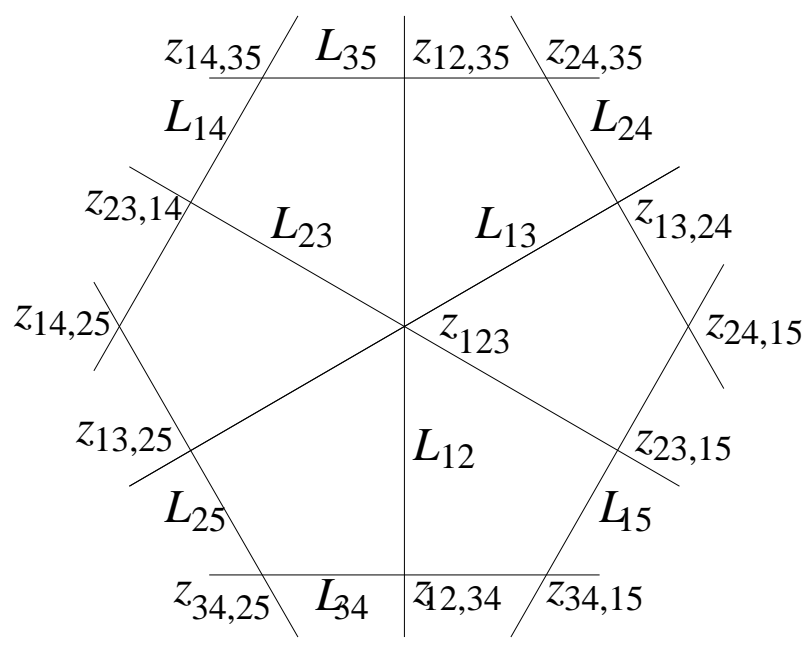

Figure 10. The line arrangement when $l>0$ and $d \leq 0$ corresponding to Mostow groups of the second type.

Then, using $d=2 p /(p-6)$, the orbifold Euler characteristic $\chi\left(\mathbf{H}_{\mathbb{C}}^{2} / \Gamma\right)$ is

$$
\begin{aligned}
\chi\left(\mathbf{H}_{\mathbb{C}}^{2} / \Gamma\right)= & \frac{1}{2 p}+\frac{1}{2 p^{2}}+\frac{1}{d p}-\frac{1}{p}-\frac{1}{p}-\frac{1}{2}-\frac{1}{p}-\frac{1}{4}-\frac{1}{2 d} \\
& \quad+\frac{1}{2}+1+1+1+\frac{1}{3}+\frac{1}{p}+\frac{1}{p}+\frac{1}{3 d}-1-1-1-1+1 \\
= & \frac{1}{2 p}+\frac{1}{2 p^{2}}+\frac{1}{2 p}-\frac{3}{p^{2}}-\frac{1}{p}-\frac{1}{4}--\frac{1}{4}+\frac{3}{2 p}+\frac{1}{3}+\frac{1}{6}-\frac{1}{p} \\
= & \frac{p-5}{2 p^{2}} .
\end{aligned}
$$

This agrees with Theorems 5.4 and 5.7 of Sauter. Note that in Theorem 5.4 Sauter is computing the volume of $\mathbf{H}_{\mathbb{C}}^{2} / \Gamma_{\mu}$ which is six times the volume of $\mathbf{H}_{\mathbb{C}}^{2} / \Gamma_{\mu \Sigma}$, which is what we have; see pages $381-383$ of [57].

5.3. Mostow groups of the second type. In this section we consider the groups with $l>0$ and $d \leq 0$. The values of $p$ and $k$ and those of $d$ and $l$ are:

\begin{tabular}{|l|rrrrrrrrrrrr|}
\hline$p$ & 3 & 3 & 3 & 3 & 3 & 4 & 4 & 4 & 5 & 5 & 6 & 6 \\
$k$ & 7 & 8 & 9 & 10 & 12 & 5 & 6 & 8 & 4 & 5 & 4 & 6 \\
$-d$ & 2 & 2 & 2 & 2 & 2 & 4 & 4 & 4 & 10 & 10 & $\infty$ & $\infty$ \\
$l$ & 42 & 24 & 18 & 15 & 12 & 20 & 12 & 8 & 20 & 10 & 12 & 6 \\
\hline
\end{tabular}

In this case the line $L_{45}$ has collapsed to a point $z_{123}$. We have drawn the line arrangement in Figure 10, which should be compared with the first figure on page 363 of Sauter [57].

A fundamental domain for these groups was constructed by Deraux, Falbel and Paupert [15]. We describe this fundamental domain. From one point of view, it may once again be obtained from the fundamental domain constructed in Section 5.1 by truncating various vertices. However, this process is more complicated and it is perhaps easier to make a direct construction. In order to emphasise the similarities with the previous two fundamental domains we try to use similar notation. 

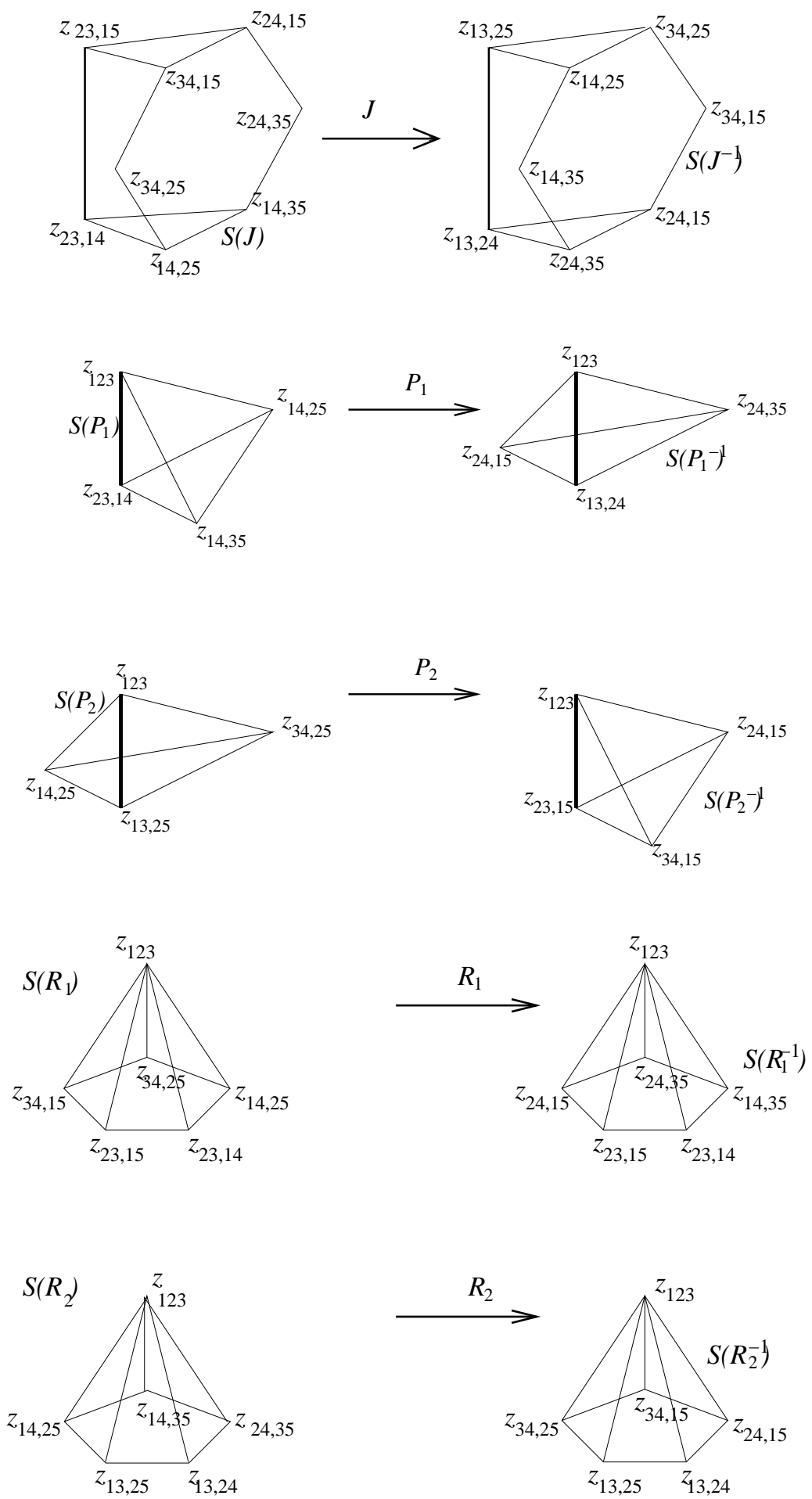

Figure 11. The side pairings. 
The polyhedron $D$ in the construction of Deraux, Falbel and Paupert [15] has ten sides. Of these, six are contained in a bisector, but the remaining four are not. The vertex $z_{234}$ has been truncated to form a triangle and so gives rise to three new vertices $z_{23,15}, z_{34,15}$ and $z_{24,15}$. These are all contained in $L_{15}$, the fixed complex line of $A_{1}^{\prime}=\left(J^{-1} R_{1}^{-1} J^{-1}\right)^{2}=J^{-1} R_{1}^{-1} R_{2}^{-1}$. There is a new side $S\left(P_{2}^{-1}\right)$ obtained by taking a cone over this triangle to $z_{123}$. Similarly, the vertex $z_{134}$ is truncated to give a triangle with vertices $z_{13,25}, z_{14,25}, z_{34,25}$. There is a side that is the cone over this triangle to $z_{123}$. This side is $S\left(P_{2}\right)$. The side pairing map $P_{2}=R_{2} R_{1}$ sends $S\left(P_{2}\right)$ to $S\left(P_{2}^{-1}\right)$. This truncation process has a further effect. Consider the triangle with vertices $z_{234}, z_{134}, z_{124}$ from Figure 7 . Because $J$ maps this triangle to itself and cyclically permutes the vertices, when we truncate the vertices $z_{234}$ and $z_{134}$ to replace them with an edge, we must also replace $z_{124}$ with an edge. Hence this triangle gets replaced with a hexagon. Likewise, the triangle with vertices $z_{234}$, $z_{134}$ and $z_{23,14}$ in Figure 7 is replaced by a pentagon. Thus the side $S\left(R_{1}\right)$ is now a cone over a pentagon. This is not contained in a bisector. The same is true of $S\left(R_{1}^{-1}\right), S\left(R_{2}\right)$ and $S\left(R_{2}^{-1}\right)$.

To summarise, the side pairings are $J, P_{1}, P_{2}, R_{1}$ and $R_{2}$ and the cycle relations are:

\begin{tabular}{|l|l|l|}
\hline Face & Cycle element & Cycle relation \\
\hline$F\left(J, J^{-1}\right)=S(J) \cap S\left(J^{-1}\right)$ & $J$ & $J^{3}=1$ \\
$F\left(J, R_{1}\right)=S(J) \cap S\left(R_{1}\right)$ & $J^{-1} R_{2}^{-1} J R_{1}$ & $J^{-1} R_{2}^{-1} J R_{1}=1$ \\
$F\left(J, P_{1}\right)=S(J) \cap S\left(P_{1}\right)$ & $J^{-1} P_{1}$ & $\left(J^{-1} P_{1}\right)^{k}=1$ \\
$F\left(J, P_{2}^{-1}\right)=S(J) \cap S\left(P_{2}^{-1}\right)$ & $J^{-1} P_{2}^{-1}$ & $\left(J^{-1} P_{2}\right)^{l}=1$ \\
$F\left(R_{1}, R_{1}^{-1}\right)=S\left(R_{1}\right) \cap S\left(R_{1}^{-1}\right)$ & $R_{1}$ & $R_{1}^{p}$ \\
$F\left(R_{2}, R_{2}^{-1}\right)=S\left(R_{2}\right) \cap S\left(R_{2}^{-1}\right)$ & $R_{2}$ & $R_{2}^{p}$ \\
$F\left(P_{1}, R_{1}\right)=S\left(P_{1}\right) \cap S\left(R_{1}\right)$ & $P_{1}^{-1} R_{2}^{-1} P_{1} R_{1}$ & $P_{1}^{-1} R_{2}^{-1} P_{1} R_{1}=1$, \\
$F\left(P_{2}^{-1}, R_{1}\right)=S\left(P_{2}^{-1}\right) \cap S\left(R_{1}\right)$ & $P_{2} R_{2}^{-1} P_{2}^{-1} R_{1}$ & $P_{2} R_{2}^{-1} P_{2}^{-1} R_{1}=1$ \\
$F\left(P_{1}, R_{2}\right)=S\left(P_{1}\right) \cap S\left(R_{2}\right)$ & $R_{2}^{-1} R_{1}^{-1} P_{1}$ & $R_{2}^{-1} R_{1}^{-1} P_{1}=1$ \\
$F\left(P_{2}, R_{1}\right)=S\left(P_{2}\right) \cap S\left(R_{1}\right)$ & $R_{1}^{-1} R_{2}^{-1} P_{2}$ & $R_{1}^{-1} R_{2}^{-1} P_{2}=1$ \\
\hline
\end{tabular}

From this we obtain a geometrical presentation for the group:

$$
\Gamma=\left\langle J, P_{1}, P_{2}, R_{1}, R_{2}: \begin{array}{c}
J^{3}=R_{1}^{p}=R_{2}^{p}=\left(P_{1}^{-1} J\right)^{k}=\left(P_{2} J\right)^{l}=I \\
P_{1}=R_{1} R_{2}, \quad P_{2}=R_{2} R_{1} \\
R_{2}=P_{1} R_{1} P_{1}^{-1}=P_{2}^{-1} R_{1} P_{2}=J R_{1} J^{-1}
\end{array}\right\rangle .
$$

Writing $P_{1}^{-1} J=A_{1}$ and $J^{-1} P_{2}^{-1}=A_{1}^{\prime}$ we obtain

$$
\left\langle\begin{array}{ll} 
& J^{3}=R_{1}^{p}=A_{1}^{k}=A_{1}^{\prime l}=1 \\
& \left.A_{1}=\left(J R_{1}^{-1} J\right)^{2}, A_{1}^{\prime}=\left(J^{-1} R_{1}^{-1} J^{-1}\right)^{2},\right\rangle \\
& A_{1} R_{1}=R_{1} A_{1}, A_{1}^{\prime} R_{1}=R_{1} A_{1}^{\prime}
\end{array}\right.
$$

We can use Propositions 4.4 and 4.5 to describe the stabiliser of $z_{123}$. The stabiliser of each other vertex is the direct product of a pair of cyclic groups. Using 
this fundamental domain, we compute the Euler characteristic as before:

\begin{tabular}{|c|c|c|}
\hline Orbit & Stabiliser & Order \\
\hline$z_{23,14}, z_{13,24}$ & $\left\langle R_{1}, A_{1}\right\rangle$ & $k p$ \\
\hline$z_{23,15}, z_{13,25}$ & $\left\langle R_{1}, A_{1}^{\prime}\right\rangle$ & $l p$ \\
\hline$z_{14,25}, z_{14,35}, z_{24,35}, z_{24,15}, z_{34,15}, z_{34,25}$ & $\left\langle A_{1}, A_{2}^{\prime}\right\rangle$ & $k l$ \\
\hline$z_{123}$ & $\left\langle R_{1}, R_{2}\right\rangle$ & \\
\hline$\left(z_{23,15}, z_{123}\right),\left(z_{13,25}, z_{123}\right)$ & $\left\langle R_{1}\right\rangle$ & $p$ \\
\hline$\left(z_{23,14}, z_{123}\right),\left(z_{13,24}, z_{123}\right)$ & $\left\langle R_{1}\right\rangle$ & $p$ \\
\hline$\left\{\left(z_{14,25}, z_{123}\right),\left(z_{14,35}, z_{123}\right),\left(z_{24,35}, z_{123}\right)\right.$, & & 1 \\
\hline$\left(z_{24,15}, z_{123}\right),\left(z_{34,15}, z_{123}\right),\left(z_{34,25}, z_{123}\right)$ & $\left\langle(I R)_{1}\right\rangle$ & \\
\hline$\left(z_{34,15}, z_{34,25}\right),\left(z_{14,25}, z_{14,35}\right),\left(z_{24,35}, z_{24,15}\right)$ & $\begin{array}{l}\left\langle J R_{1}\right\rangle \\
\left\langle J R^{-1}\right\rangle\end{array}$ & $\begin{array}{l}2 k \\
2 l\end{array}$ \\
\hline$\left(z_{14,35}, z_{24,35}\right),\left(z_{24,15}, z_{34,15}\right),\left(z_{34,25}, z_{14,25}\right)$ & $\begin{array}{l}\left\langle J R_{1}^{-1}\right\rangle \\
\left\langle R_{1}\right\rangle\end{array}$ & $2 l$ \\
\hline$\left(z_{23,14}, z_{23,15}\right),\left(z_{13,24}, z_{13,25}\right)$ & $\left\langle R_{1}\right\rangle$ & $p$ \\
\hline$\left\{\begin{array}{l}\left(z_{23,14}, z_{14,25}\right), \\
\left(z_{23,14}, z_{14,35}\right), \\
\left(z_{13,24}, z_{24,15}\right),\left(z_{13,24}, z_{24,35}\right)\end{array}\right\}$ & $\left\langle A_{1}\right\rangle$ & $k$ \\
\hline$\left\{\begin{array}{l}\left(z_{23,15}, z_{24,15}\right),\left(z_{23,15}, z_{34,15}\right) \\
\left(z_{13,25}, z_{14,25}\right),\left(z_{13,25}, z_{34,25}\right)\end{array}\right\}$ & & \\
\hline$F\left(J, P_{1}\right), F\left(J^{-1}, P-1^{-1}\right)$ & $\left\langle A_{1}\right\rangle$ & $k$ \\
\hline$F\left(J, P_{2}^{-1}\right), F\left(J^{-1}, P_{2}\right)$ & $\left\langle A_{1}^{\prime}\right\rangle$ & $l$ \\
\hline$F\left(R_{1}, R_{1}^{-1}\right)$ & $\left\langle R_{1}\right\rangle$ & $p$ \\
\hline$F\left(R_{2}, R_{2}^{-1}\right)$ & $\left\langle R_{2}\right\rangle$ & $p$ \\
\hline$F\left(J, J^{-1}\right)$ & $\langle J\rangle$ & 3 \\
\hline$F\left(J, R_{1}\right), F\left(J, R_{1}^{-1}\right), F\left(J^{-1}, R_{2}\right), F\left(J^{-1}, R_{2}^{-1}\right)$ & 1 & 1 \\
\hline$F\left(P_{1}, R_{1}\right), F\left(P_{1}, R_{1}^{-1}\right), F\left(P_{1}^{-1}, R_{2}\right), F\left(P_{1}^{-1}, R_{2}^{-1}\right)$ & 1 & 1 \\
\hline$F\left(P_{2}^{-1}, R_{1}\right), F\left(P_{2}^{-1}, R_{1}^{-1}\right), F\left(P_{2}, R_{2}\right), F\left(P_{2}, R_{2}^{-1}\right)$ & 1 & 1 \\
\hline$F\left(P_{1}, R_{2}\right), F\left(P_{1}^{-1}, R_{1}^{-1}\right), F\left(R_{1}, R_{2}^{-1}\right)$ & 1 & 1 \\
\hline$F\left(P_{2}, R_{1}\right), F\left(P_{2}^{-1}, R_{2}^{-1}\right), F\left(R_{2}, R_{1}^{-1}\right)$ & 1 & 1 \\
\hline$S(J) S\left(J^{-1}\right)$ & 1 & 1 \\
\hline$S\left(P_{1}\right), S\left(P_{1}^{-1}\right)$ & 1 & 1 \\
\hline$S\left(P_{2}\right), S\left(P_{2}^{-1}\right)$ & 1 & 1 \\
\hline$S\left(R_{1}\right), S\left(R-1^{-1}\right)$ & 1 & 1 \\
\hline$S\left(R_{2}\right), S\left(R_{2}^{-1}\right)$ & 1 & 1 \\
\hline$D$ & 1 & 1 \\
\hline
\end{tabular}

Then the orbifold Euler characteristic $\chi\left(\mathbf{H}_{\mathbb{C}}^{2} / \Gamma\right)$ is

$$
\begin{aligned}
\chi\left(\mathbf{H}_{\mathbb{C}}^{2} / \Gamma\right)= & \frac{1}{k p}+\frac{1}{l p}+\frac{1}{k l}+\frac{1}{6 d^{2}}-\frac{1}{p}-\frac{1}{p}-1-\frac{1}{2 k}-\frac{1}{2 l}-\frac{1}{p}-\frac{1}{k}-\frac{1}{l} \\
& +\frac{1}{k}+\frac{1}{l}+\frac{1}{p}+\frac{1}{p}+\frac{1}{3}+1+1+1+1+1-1-1-1-1-1+1 \\
= & \frac{1}{k p}+\frac{1}{l p}+\frac{1}{k l}+\frac{1}{24}-\frac{1}{2 p}+\frac{3}{2 p^{2}}-\frac{1}{2 k}-\frac{1}{2 l}-\frac{1}{p}+\frac{1}{3} \\
= & \frac{1}{2}\left(\frac{1}{2}-\frac{1}{p}\right)^{2}+\frac{1}{k}\left(\frac{1}{2}-\frac{1}{p}-\frac{1}{k}\right)
\end{aligned}
$$

where we have used $1 / l=1 / 2-1 / p-1 / k$ on the last line. This agrees with Theorem 5.1 ' of Sauter. 
5.4. The Gauss-Picard modular group. In this section we briefly discuss the group with $p=\infty$ and $k=4$. This group is an index two subgroup of the GaussPicard modular group $\mathrm{PU}\left(2,1 ; \mathcal{O}_{1}\right)$; see Example 4.6 .6 and Section 5.3 of Holzapfel [29], also Parker [46], Francsics and Lax [17] and Falbel, Francsics, Parker [16]. In this case $k=l$ and the extra holomorphic symmetry of order 2 , denoted $Q$, has the effect of swapping the cone points $v_{2}$ and $v_{3}$ and swapping $v_{4}$ and $v_{5}$. Therefore, $Q R_{1} Q^{-1}=R_{1}, Q R_{2} Q^{-1}=R_{3}$ and $Q J Q^{-1}=J^{-1}$.

In [16] the standard off-diagonal Hermitian form was used. In this case $L_{23}$ has collapsed to the point infinity in the Siegel domain model of $\mathbf{H}_{\mathbb{C}}^{2}$. The matrices become:

$$
\begin{array}{ll}
J=\left[\begin{array}{ccc}
0 & 0 & 1 \\
0 & 1 & -1-i \\
1 & 1-i & -1
\end{array}\right], \quad R_{1}=\left[\begin{array}{lll}
1 & 0 & i \\
0 & 1 & 0 \\
0 & 0 & 1
\end{array}\right], \\
Q=\left[\begin{array}{ccc}
1 & 1-i & -1 \\
0 & -1 & 1+i \\
0 & 0 & 1
\end{array}\right], \\
A_{1}=\left[\begin{array}{ccc}
-i & -1-i & 1+i \\
0 & 1 & -1-i \\
0 & 0 & -i
\end{array}\right], A_{1}^{\prime}=\left[\begin{array}{ccc}
-i & -1+i & 1+i \\
0 & 1 & 1-i \\
0 & 0 & -i
\end{array}\right] .
\end{array}
$$

Francsics and Lax [18] construct the Ford domain for this group and Falbel, Francsics and Parker [16] use Poincaré's theorem to give a presentation of the group. In our notation, this is:

$$
\left\langle J, R_{1}, Q: \begin{array}{l}
(J Q)^{2}=Q^{2}=J^{3}=\left(R_{1} J Q\right)^{12}=\left(J R_{1}\right)^{8}=1 \\
\left(J Q R_{1}\right)^{3} R_{1}=R_{1}\left(J Q R_{1}\right)^{3}, Q R_{1}=R_{1} Q
\end{array}\right\rangle
$$

Taking the index two subgroup generated by $J$ and $R_{1}$ and substituting for $A_{1}, A_{1}^{\prime}$ and $P$, the Deligne-Mostow group with $p=\infty$ and $k=4$ has presentation

$$
\left\langle\begin{array}{ll} 
& J^{3}=A_{1}^{4}=A_{1}^{\prime 4}=\left(J A_{1}^{-1}\right)^{6}=1 \\
& A_{1}=\left(J R_{1}^{-1} J\right)^{2}, A_{1}^{\prime}=\left(J^{-1} R_{1}^{-1} J^{-1}\right)^{2} \\
& A_{1} R_{1}=R_{1} A_{1}, A_{1}^{\prime} R_{1}=R_{1} A_{1}^{\prime}
\end{array}\right\rangle .
$$

\section{Problems}

I will conclude this article by giving a list of questions that, as far as I know, are open. I expect these to be of varying levels of difficulty. In particular, the first problem is fundamental, it is probably difficult and its solution would be of widespread interest.

Problem 6.1. Do there exist non-arithmetic lattices in $\mathrm{PU}(n, 1)$ for $n \geq 4$ ?

My guess (which I would not want to state as a conjecture) is that nonarithmetic lattices exist in all dimensions. If this were so, then a possible reason why such lattices have not yet been found is that we have very few methods of constructing lattices.

Problem 6.2. Give a purely geometric description, for example via line arrangements or the construction a fundamental domain, for the group associated to Mumford's fake projective plane.

Problem 6.3. Find the precise relationship between Deraux's lattice and the fake projective plane constructed by Prasad and Yeung over the same number field. 
PROBLEM 6.4. Interpret the commensurability theorems in terms of fundamental domains. Thereby give fundamental domains and presentations for other Deligne-Mostow groups.

Problem 6.5. Give fundamental domains for the Deligne-Mostow groups without three fold symmetry, particularly those that are not commensurable with one that does have three fold symmetry.

Problem 6.6. Construct fundamental domains for lattices in higher complex dimension.

\section{References}

[1] R. Alezais; Sur une classe de fonctions hyperfuchsiennes. Ann. École Norm. Sup 19 (1902), 261-323.

[2] D. Allcock; The Leech lattice and complex hyperbolic reflections. Invent. Math. 140 (2000) 283-301.

[3] D. Allcock; New complex- and quaternion-hyperbolic reflection groups. Duke Math. J. 103 (2000) 303-333.

[4] D. Allcock, J.A. Carlson \& D. Toledo; The complex hyperbolic geometry of the moduli space of cubic surfaces. J. Algebraic Geometry 11 (2002) 659-724.

[5] D. Allcock, J.A. Carlson \& D. Toledo; The moduli space of cubic threefolds as a ball quotient. Memoirs of the A.M.S. (to appear).

[6] G. Barthel, F. Hirzebruch \& T. Höfer; Geradenkonfigurationen und Algebraische Flächen. Aspects of Mathematics, Vieweg 1987.

[7] J.S. Birman; Braids, Links and Mapping Class Groups; Annals of Maths. Studies 82, Princeton University Press (1974).

[8] R.K. Boadi; Lattices in Complex Hyperbolic Space. PhD Thesis, KNUST, Kumasi (in preparation).

[9] A. Borel \& Harish-Chandra; Arithmetic subgroups of algebraic groups. Annals of Maths $\mathbf{7 5}$ (1962) 485-535.

[10] K. Corlette; Archimedean superrigidity and hyperbolic geometry. Annals of Maths $\mathbf{1 3 5}$ (1992) 165-182.

[11] P. Deligne \& G.D. Mostow; Monodromy of hypergeometric functions and non-lattice integral monodromy. Publ. Math. I.H.E.S. 63 (1986), 5-89.

[12] P. Deligne \& G.D. Mostow; Commensurability Among Lattices in PU $(1, n)$. Annals of Maths. Studies 132, Princeton University Press (1993).

[13] M. Deraux; Dirichlet domains for the Mostow lattices. Experimental Maths. 14 (2005), $467-490$.

[14] M. Deraux; Deforming the $\mathbb{R}$-Fuchsian $(4,4,4)$-triangle group into a lattice. Topology 45 (2006) 989-1020.

[15] M. Deraux, E. Falbel \& J. Paupert; New constructions of fundamental polyhedra in complex hyperbolic space. Acta Mathematica 194 (2005), 155-201.

[16] E. Falbel, G. Francsics \& J.R. Parker; The geometry of Picard modular groups. Preprint.

[17] E. Falbel \& J.R. Parker; The geometry of the Eisenstein-Picard modular group. Duke Math. J 131 (2006), 249-289.

[18] G. Francsics \& P.D. Lax; An explicit fundamental domain for the Picard modular group in two complex dimensions. Proc. Nat. Acad. Sci. USA 103 (2006), 11103-11105.

[19] G. Giraud, Sur certaines fonctions automorphes de deux variables. Ann. Sci. École Norm. Sup. 38 (1921), 43-164.

[20] W.M. Goldman; Complex Hyperbolic Geometry. Oxford University Press, 1999.

[21] M. Gromov \& I. Piatetski-Shapiro; Nonarithmetic groups in Lobachevsky spaces. Publ. I.H.E.S. 66 (1988) 93-103.

[22] M. Gromov \& R. Schoen; Harmonic maps into singular spaces and p-adic superrigidity for lattices in groups of rank one. Publ. I.H.E.S. 76 (1992) 165-246.

[23] F. Hirzebruch; Some examples of algebraic surfaces. Papers in Algebra, Analysis and Statistics, ed: R. Lidl. Contemporary Mathematics 9, A.M.S. 1981. 
[24] F. Hirzebruch; Arrangements of lines and algebraic surfaces. Arithmetic and Geometry Vol II, 113-140, ed: M. Artin \& J. Tate, Progress in Mathematics 36, Birkhäuser, 1983.

[25] F. Hirzebruch; Chern numbers of algebraic surfaces: an example. Math. Ann. 266 (1984) 351-356.

[26] R.-P. Holzapfel; Invariants of arithmetic ball quotient surfaces. Math. Nachr. 103 (1981), $117-153$.

[27] R.-P. Holzapfel; Geometry and Arithmetic Around Euler Partial Differential Equations. Reidel, Dordrecht (1986).

[28] R.-P. Holzapfel; Symplectic representation of a braid group on 3-sheeted covers of the Riemann sphere. Serdica Math. J. 23 (1997), 143-164.

[29] R.-P. Holzapfel; Ball and Surface Arithmetics. Aspects of Mathematics, Vieweg 1998.

[30] M. Ishida; Hirzebruch's examples of surfaces with $c_{1}^{2}=3 c_{2}$. Algebraic Geometry, ed: M. Raynaud \& T. Shioda. Lecture Notes in Mathematics 1016, Springer, 1983.

[31] M. Ishida \& F. Kato; The strong rigidity theorem for non-Archimedean uniformization. Tohoku Math. J. 50 (1998), 537-555.

[32] F. Kato; Arithmetic structure of Mumford's fake projective plane. Preprint.

[33] G. Lauricella, Sulle funzioni ipergeometiche a piu variabili. Remd. Circ. Math. Palermo 7 (1893) 111-158.

[34] R. Le Vavasseur, Sur le système d'équations aux dérivées partielles simultanées auquelles satisfait la série hypergéometrique à deux variables. J. Fac. Sci. Toulouse 7 (1896), 1-205.

[35] R.A. Livné; On Certain Covers of the Universal Elliptic Curve, Ph.D. Thesis, Harvard University, 1981.

[36] G. Margulis; Arithmeticity of the irreducible lattices in the semisimple groups of rank greater than 1. Invent. Math. 76 (1984) 93-120.

[37] C.T. McMullen; Braid groups and Hodge theory. Preprint.

[38] D.B. McReynolds; Arithmetic Lattices in $\mathrm{SU}(n, 1)$. (to appear).

[39] Y. Miyaoka; Algebraic surfaces with positive indices. Classification of Algebraic and Analytic Manifolds, 281-301, ed: K. Ueno, Progress in Mathematics 39, Birkhäuser, 1983.

[40] G.D. Mostow; Strong Rigidity of Locally Symmetric Spaces. Annals of Maths. Studies 78, Princeton University Press (1973).

[41] G.D. Mostow, On a remarkable class of polyhedra in complex hyperbolic space. Pacific Journal of Maths. 86 (1980) 171-276.

[42] G.D. Mostow; Generalized Picard lattices arising from half-integral conditions. Publ. Math. I.H.E.S. 63 (1986), 91-106.

[43] G.D. Mostow; Braids, hypergeometric functions and lattices. Bulletin of the A.M.S. 16 (1987) 225-246.

[44] G.D. Mostow; On discontinuous action of monodromy groups on the complex $n$-ball. Journal of the A.M.S. 1 (1988), 555-586.

[45] D. Mumford; An algebraic surface with $K$ ample, $\left(K^{2}\right)=9, p_{g}=q=0$. American J. Maths. 101 (1979), 233-244.

[46] J.R. Parker; On the volumes of cusped, complex hyperbolic manifolds and orbifolds. Duke Math. J. 94, No. 3 (1998), 433-464.

[47] J.R.Parker; Cone metrics on the sphere and Livné's lattices. Acta Math 196 (2006), 1-64.

[48] J.R. Parker; Unfaithful complex hyperbolic triangle groups I: Involutions. Pacific Journal of Maths 238 (2008) 145-169.

[49] J.R. Parker, Complex Hyperbolic Kleinian Groups. Cambridge University Press (to appear).

[50] J.R. Parker \& J. Paupert, Unfaithful complex hyperbolic triangle groups II: Higher order reflections. Pacific Journal of Maths 239 (2009) 357-389.

[51] J.R. Parker \& J.M. Thompson; A fundamental domain for Deraux's lattice. (in preparation).

[52] É. Picard, Sur une extension aux fonctions de deux variables indépendentes analogues aux fonctions modulaires. Acta Math. 2 (1883), 114-135.

[53] É. Picard; Sur des formes quadratiques ternaires indéfinies indéterminées conjuguées et sur les fonctions hyperfuchsiennes correspondantes. Acta Math. 5 (1884), 121-182.

[54] É. Picard, Sur les fonctions hyperfuchsiennes provenant des séries hypergéométriques de deux variables. Ann. Sci. École Norm.Sup. 62 (1885), 357-384.

[55] G. Prasad \& S.-K. Yeung; Fake projective planes; Invent. Math. 168 (2007), 321-370.

[56] M.S. Raghunathan; Discrete Subgroups of Lie Groups. Springer 1972. 
[57] J.K. Sauter; Isomorphisms among monodromy groups and applications to lattices in PU(1,2). Pacific J. Maths. 146 (1990), 331-384.

[58] R.E. Schwartz; Spherical CR Geometry and Dehn Surgery. Annals of Maths. Studies 165, Princeton University Press (2007)

[59] H.A. Schwarz; Über diejenige Fälle, in welchen die Gaussische hypergeometrische Reihe eine algebraische Funktion ihres vierten Elementes darstellt. J. reine angew. Math. 75 (1873) 292-335.

[60] O.V. Shvartsman; Discrete reflection groups in the complex ball. Funktsional. Anal. i Prilozhen 18 (1984) 88-89. Translated in: Functional Analysis and its Applications 18 (1984) 81-83.

[61] O.V. Shvartsman; An example of a nonarithmetic discrete group in the complex ball. Lie groups, their discrete subgroups and invariant theory, ed E.B. Vinberg; Advances in Soviet Mathematics 8 (1992) 191-196.

[62] T. Terada; Fonctions hypergéométriques $F_{1}$ et fonctions automorphes I. J. Math. Soc. Japan 35 (1983) 451-475.

[63] W.P. Thurston; Shapes of polyhedra and triangulations of the sphere. Geometry and Topology Monographs 1 (1998), The Epstein Birthday Schrift, 511-549, ed: I. Rivin, C. Rourke \& C. Series.

[64] D. Toledo; Maps between complex hyperbolic surfaces. Geometriae Dedicata 97 (2003), $115-128$.

[65] W.A. Veech; Flat surfaces. American J. Maths 115 (1993) 589-689.

[66] M. Weber; Fundamentalbereiche komplex hyperbolische Flächen. Bonner Mathematische Schriften 254, 1993.

[67] T. Yamazaki \& M. Yoshida; On Hirzebruch's examples of surfaces with $c_{1}^{2}=3 c_{2}$. Math. Ann. 266 (1984) 421-431.

[68] S.T. Yau; Calabi's conjecture and some new results in algebraic geometry. Proc. Nat. Acad. Sci, U.S.A. 74 (1977) 1798-1799.

[69] M. Yoshida; Volume formula for certain discrete reflection groups in PU(2,1). Mem. Fac Sci Kyushu Univ 36 (1982) 1-11.

[70] M. Yoshida; Graphs attached to certain complex hyperbolic discrete reflection groups. Topology 25 (1986) 175-187.

Department of Mathematical Sciences,, University of Durham,, South Road,, Durham DH1 3LE, ENGLAND., E-MAIL: j.r.parker@durham.ac.uk 